\title{
Oscillations of Dirac and Majorana neutrinos in matter and a magnetic field
}

\author{
Maxim Dvornikov $a, b, \circledast$ and Jukka Maalampi ${ }^{a, \text {, }}$ \\ ${ }^{a}$ Department of Physics, P.O. Box 35, FIN-40014 University of Jyväskylä, Finland; \\ ${ }^{b}$ Departamento de Física y Centro de Estudios Subatómicos, \\ Universidad Técnica Federico Santa María, Casilla 110-V, Valparaíso, Chile; \\ ${ }^{c}$ IZMIRAN, 142190, Troitsk, Moscow Region, Russia; \\ ${ }^{d}$ Helsinki Institute of Physics, P.O. Box 64, FIN-00014 University of Helsinki, Finland
}

(Dated: December 11, 2018)

\begin{abstract}
We study the evolution of massive mixed Dirac and Majorana neutrinos in matter under the influence of a transversal magnetic field. The analysis is based on relativistic quantum mechanics. We solve exactly the evolution equation for relativistic neutrinos, find the neutrino wave functions, and calculate the transition probability for spin-flavor oscillations. We analyze the dependence of the transition probability on the external fields and compare the cases of Dirac and Majorana neutrinos. The evolution of Majorana particles in vacuum is also studied and correction terms to the standard oscillation formula are derived and discussed. As a possible application of our results we discuss the spin-flavor transitions in supernovae.
\end{abstract}

PACS numbers: 14.60.Pq, 14.60.St, 03.65.Pm

Keywords: neutrino oscillations, background matter, magnetic field, supernova neutrinos

\section{INTRODUCTION}

If massive neutrinos possess nonzero transition magnetic moments, transitions $\nu_{\beta}^{\mathrm{L}} \leftrightarrow \nu_{\alpha}^{\mathrm{R}}$ that change both spin and flavor of neutrinos can happen in electromagnetic fields. Such transitions may be realized, e.g., in astrophysical environments where strong magnetic fields are present. Oscillations of Dirac neutrinos in an external magnetic field were studied in Ref. [1]. In this scenario one has a transition to the sterile neutrino state $\nu_{\mathrm{R}}$. While considering oscillations of Majorana neutrinos with transition magnetic moments in an external magnetic field (see, e.g., Refs. [2, 3, 4] ), one has transitions between active neutrino states since $\nu_{\alpha}^{\mathrm{R}}=\left(\nu_{\alpha}^{\mathrm{L}}\right)^{c}$ for a Majorana particle.

Neutrino spin-flavor oscillations in solar magnetic fields have been earlier studied in connection to the solar neutrino problem (see, e.g., Ref. [5]). It was thought that this neutrino oscillations channel could explain at least partly the deficit of the electron neutrinos in the measured solar neutrino flux. It is now clear that such spin-flavor oscillations cannot play any significant role and the deficit can be satisfactorily explained in terms of active-active conversions and the Mikheyev-Smirnov-Wolfenstein matter effect $[\underline{6}]$.

The influence of strong magnetic fields of neutron stars on neutrino oscillations was studied in Refs. [7, 8, 9]. It was shown that spin-flavor transition may have important effects in the neutron star environment. In these investigations neutrino spin-flavor oscillations were described for realistic profiles of matter densities and magnetic fields and the appearance of resonances in neutrino oscillations was examined. For further details on the neutrino oscillations in electromagnetic fields as well as neutrino electromagnetic properties the reader is referred to the recent review [10].

In this paper we shall return to the question of the spinflavor oscillations using an approach that differs from the one usually followed. It is usual to describe neutrino oscillations, including the spin-flavor transitions, on the basis of the quantum mechanical evolution equation. Instead of this Schrödinger picture, we will apply relativistic quantum mechanical picture based on the Dirac theory. In this approach, we will study spin-flavor oscillations of Dirac and Majorana neutrinos in matter and in an external magnetic field, extending our earlier use of the method [11, 12, 13, 14] to this new problem. We should also mention that the majority of the previous studies of neutrino spin-flavor oscillations are restricted to the case of Majorana neutrinos. We will investigate both the Dirac and Majorana cases as the nature of neutrinos is still an open question 15 .

The plan of this paper is as follows. We will start by writing down the relativistic wave equations that take into account the background matter and external magnetic field. We formulate the initial condition problem for these systems (see also Refs. 11, 12, 13, 14]). We then derive for both Dirac and Majorana neutrinos a Hamiltonian analogous to that of the standard quantum mechanical approach and solve exactly the resulting evolution equation for relativistic neutrinos. We then analyze the behavior of the transition probability for Dirac and Majorana neutrinos at various magnetic field strengths and matter densities. Our results will be summarized and their applications to some astrophysical situations discussed in Sec. V].

*Electronic address: maxim.dvornikov@usm.cl

${ }^{\dagger}$ Electronic address: maalampi@cc.jyu.fi 


\section{EVOLUTION OF DIRAC NEUTRINOS IN MATTER AND TRANSVERSAL MAGNETIC FIELD}

Let us study the evolution of two neutrino flavor states $\nu_{\lambda}, \lambda=\alpha, \beta$, in a nonmoving and unpolarized matter under the influence of an external magnetic field. We assume that the mass eigenstates $\psi_{a}, a=1,2$, are related to the flavor eigenstate neutrinos through the transformation

$$
\nu_{\lambda}=\sum_{a=1,2} U_{\lambda a} \psi_{a}, \quad\left(U_{\lambda a}\right)=\left(\begin{array}{cc}
\cos \theta & -\sin \theta \\
\sin \theta & \cos \theta
\end{array}\right),
$$

where $\theta$ is a mixing angle. In this section we shall assume that the mass eigenstates are Dirac particles. We set the following initial conditions (see also Refs. [11, 12, 13, 14]):

$$
\nu_{\alpha}(\mathbf{r}, 0)=0, \quad \nu_{\beta}(\mathbf{r}, 0)=\nu_{\beta}^{(0)} e^{\mathrm{ikr}}
$$

where $\mathbf{k}=(k, 0,0)$ is the initial momentum and $\nu_{\beta}^{(0) \mathrm{T}}=$ $(1 / 2)(1,-1,-1,1)$ (see below). This corresponds to a relativistic neutrino of the flavor $\beta$, with its spin directed oppositely to the particle momentum, i.e. a left-handed neutrino $\nu_{\beta}^{\mathrm{L}}$. The system is taken not to contain the other neutrino flavor $\nu_{\alpha}$ initially.

Note that from the physical point of view it would be more realistic to choose a localized in space initial condition rather than that in Eq. (2.2). It is well known, however, that from the point of view of oscillations the plane wave and wave packet approaches in practice lead to equivalent results [16]. Moreover the initial condition problem (2.2) for the cases of flavor and spin-flavor oscillations in various external fields was solved in our previous publications [11, 12, 13, 14].

The Dirac equation for the mass eigenstates wave functions is of the form (see Refs. 13, 14]),

$$
\mathrm{i} \dot{\psi}_{a}=\mathcal{H}_{a} \psi_{a}+\left(V_{m}+V_{B}\right) \psi_{b}, \quad a \neq b,
$$

where $\mathcal{H}_{a}=(\boldsymbol{\alpha} \mathbf{p})+\beta m_{a}+g_{a}\left(1-\gamma_{5}\right) / 2$ is the massdiagonal part of the Hamiltonian, $m_{a}$ are the masses associated with the states $\psi_{a}$, and $\boldsymbol{\alpha}=\gamma^{0} \gamma, \beta=\gamma^{0}$, and $\boldsymbol{\Sigma}=$ $\gamma^{5} \gamma^{0} \gamma$ are Dirac matrices. The term $V_{m}=g\left(1-\gamma_{5}\right) / 2$ describes the interaction of neutrinos with particles in the background matter. The matrix $\left(g_{a b}\right)$, given below, is not diagonal in the mass eigenstate basis. The term $V_{B}=-\mu B \beta \Sigma_{3}$ is the energy operator associated with the interactions of neutrinos with the magnetic field, and the magnetic moment matrix $\left(\mu_{a b}\right)$ is, like $\left(g_{a b}\right)$, in general nondiagonal. The magnetic field is taken to be transversal with respect to the initial neutrino momentum, i.e., $\mathbf{B}=(0,0, B)$. The $V_{m}$ and $V_{B}$ terms are responsible for the possible mixing between different neutrino mass eigenstates.

The matrix $\left(g_{a b}\right)$ that describes the interactions of neutrinos with matter is in the mass eigenstates basis of the form

$$
\begin{aligned}
g_{a b} & =\sum_{\lambda=\alpha \beta} U_{a \lambda}^{\dagger} f_{\lambda} U_{\lambda b} \\
& =\left(\begin{array}{cc}
f_{\alpha} \cos ^{2} \theta+f_{\beta} \sin ^{2} \theta & -\sin \theta \cos \theta\left[f_{\alpha}-f_{\beta}\right] \\
-\sin \theta \cos \theta\left[f_{\alpha}-f_{\beta}\right] & f_{\alpha} \sin ^{2} \theta+f_{\beta} \cos ^{2} \theta
\end{array}\right) .
\end{aligned}
$$

We will denote $g_{a a}=g_{a}$ and $g_{12}=g_{21}=g$. If we identify the flavor $\alpha$ as $\equiv \nu_{\mu}$ or $\nu_{\tau}$ and the flavor $\beta$ as $\equiv \nu_{e}$, the effective potentials $f_{\lambda}, \lambda=\alpha, \beta$, are given by (see Ref. [17]):

$$
\begin{aligned}
f_{\lambda} & =\sqrt{2} G_{\mathrm{F}} \sum_{f=e, p, n} n_{f} q_{f}^{(\lambda)}, \\
q_{f}^{(\alpha)} & =\left(I_{3 \mathrm{~L}}^{(f)}-2 Q^{(f)} \sin ^{2} \theta_{W}\right), \\
q_{f}^{(\beta)} & =\left(I_{3 \mathrm{~L}}^{(f)}-2 Q^{(f)} \sin ^{2} \theta_{W}+\delta_{e f}\right),
\end{aligned}
$$

where $n_{f}$ is the number density, $I_{3 \mathrm{~L}}^{(f)}$ the third isospin component and $Q^{(f)}$ the electric charge of the background particle of the type $f, \theta_{W}$ is the weak mixing angle, and $G_{F}$ is the Fermi constant. It is assumed in Eq. (2.5) that matter consists of electrons, protons, and neutrons and that it is unpolarized and at rest.

As to the interactions of neutrinos with the magnetic field, we have assumed when writing Eq. (2.3) that the magnetic moment matrix in the mass eigenstates basis is antidiagonal, i.e., $\mu_{a a}=0$ and $\mu_{12}=\mu_{21}=\mu \neq 0$. Magnetic moment matrices of this type were studied in our previous works [13, 14]. The situation with the values of Dirac neutrino magnetic moments is disputable [18]; however, the existence of large off-diagonal magnetic moments of Dirac neutrinos is not excluded. Let us note that the solution to the Dirac-Pauli equation for a neutrino with $\mu_{a a} \neq 0$ propagating in an arbitrary moving and polarized medium was recently obtained in Ref. [19]. However in that work the case of only one neutrino flavor was studied. 14],

The general solution of Eq. (2.3) has the form [12, 13,

$$
\begin{aligned}
\psi_{a}(\mathbf{r}, t)= & \exp \left(-\mathrm{i} g_{a} t / 2\right) \int \frac{\mathrm{d}^{3} \mathbf{p}}{(2 \pi)^{3 / 2}} e^{\mathrm{i} \mathbf{p r}} \\
& \times \sum_{\zeta= \pm 1}\left[a_{a}^{(\zeta)}(t) u_{a}^{(\zeta)} \exp \left(-\mathrm{i} E_{a}^{(\zeta)} t\right)\right. \\
& \left.+b_{a}^{(\zeta)}(t) v_{a}^{(\zeta)} \exp \left(+\mathrm{i} E_{a}^{(\zeta)} t\right)\right]
\end{aligned}
$$

where the energies are given by (see, e.g., Ref. [20])

$$
E_{a}^{(\zeta)}=\sqrt{m_{a}^{2}+\left(|\mathbf{p}|-\zeta g_{a} / 2\right)^{2}}
$$

In the relativistic limit one has

$$
E_{a}^{(\zeta)} \approx|\mathbf{p}|+\frac{m_{a}^{2}}{2|\mathbf{p}|}-\zeta \frac{g_{a}}{2}
$$

To obtain Eq. (2.8) we neglect the term $\sim g_{a}^{2}$ compared to the neutrino mass squared $m_{a}^{2}$. For the situation of neutrino propagation in the expanding envelope formed after 
the supernova explosion, which is studied below, the effective potentials are $g_{a} \sim 10^{-12}-10^{-11} \mathrm{eV}$. Accounting for the neutrino mass in the $\mathrm{eV}$ range, the approximation made is always valid.

The expressions for the basis spinors $u_{a}^{(\zeta)}$ and $v_{a}^{(\zeta)}$ can be found in Ref. 20]. When one studies the situation where the initial momentum is very large, $k \gg m_{a}$, one can neglect the neutrino mass dependence of the spinors and present them in the form

$$
\begin{gathered}
u^{+}=\frac{1}{2}\left(\begin{array}{l}
1 \\
1 \\
1 \\
1
\end{array}\right), \quad u^{-}=\frac{1}{2}\left(\begin{array}{c}
1 \\
-1 \\
-1 \\
1
\end{array}\right), \\
v^{+}=\frac{1}{2}\left(\begin{array}{c}
1 \\
1 \\
-1 \\
-1
\end{array}\right), \quad v^{-}=\frac{1}{2}\left(\begin{array}{c}
1 \\
-1 \\
1 \\
-1
\end{array}\right) .
\end{gathered}
$$

Note that the coefficients $a_{a}^{(\zeta)}(t)$ and $b_{a}^{(\zeta)}(t)$ in Eq. (2.6) are functions of time due to the presence of the nondiagonal interactions $V_{m}$ and $V_{B}$. Our main goal is to determine these coefficients so that both Eq. (2.3) and the initial condition (2.2) are satisfied.
Using Eqs. (2.3) and (2.6) and the orthonormality of the basis spinors (2.9) we arrive to the following ordinary differential equations for the coefficients $a_{a}^{(\zeta)}$ and $b_{a}^{(\zeta)}[13$, 14]:

$$
\begin{aligned}
\mathrm{i} \dot{a}_{a}^{(\zeta)}= & \exp \left[\mathrm{i}\left(g_{a}-g_{b}\right) t / 2\right] \exp \left(+\mathrm{i} E_{a}^{(\zeta)} t\right) u^{(\zeta) \dagger}\left(V_{m}+V_{B}\right) \\
& \times \sum_{\zeta^{\prime}= \pm 1}\left[a_{b}^{\left(\zeta^{\prime}\right)} u^{\left(\zeta^{\prime}\right)} \exp \left(-\mathrm{i} E_{b}^{\left(\zeta^{\prime}\right)} t\right)\right. \\
& \left.+b_{b}^{\left(\zeta^{\prime}\right)} v^{\left(\zeta^{\prime}\right)} \exp \left(+\mathrm{i} E_{b}^{\left(\zeta^{\prime}\right)} t\right)\right] \\
\mathrm{i} \dot{b}_{a}^{(\zeta)}= & \exp \left[\mathrm{i}\left(g_{a}-g_{b}\right) t / 2\right] \exp \left(-\mathrm{i} E_{a}^{(\zeta)} t\right) v^{(\zeta) \dagger}\left(V_{m}+V_{B}\right) \\
& \times \sum_{\zeta^{\prime}= \pm 1}\left[a_{b}^{\left(\zeta^{\prime}\right)} u^{\left(\zeta^{\prime}\right)} \exp \left(-\mathrm{i} E_{b}^{\left(\zeta^{\prime}\right)} t\right)\right. \\
& \left.+b_{b}^{\left(\zeta^{\prime}\right)} v^{\left(\zeta^{\prime}\right)} \exp \left(+\mathrm{i} E_{b}^{\left(\zeta^{\prime}\right)} t\right)\right] .
\end{aligned}
$$

One easily sees that $\left\langle u^{-}\left|V_{m}\right| u^{-}\right\rangle=g,\left\langle v^{+}\left|V_{m}\right| v^{+}\right\rangle=g$, $\left\langle u^{ \pm}\left|V_{B}\right| u^{\mp}\right\rangle=-\mu B$ and $\left\langle v^{ \pm}\left|V_{B}\right| u^{\mp}\right\rangle=-\mu B$. All the other scalar products in Eq. (2.10) vanish.

Let us introduce a four-component wave function $\Psi^{\prime}$ defined as $\Psi^{\prime} \mathrm{T}=\left(a_{1}^{-}, a_{2}^{-}, a_{1}^{+}, a_{2}^{+}\right)$. Then we can rewrite Eq. (2.10) in the form of a Schrödinger equation:

$$
\mathrm{i} \frac{\mathrm{d} \Psi^{\prime}}{\mathrm{d} t}=H^{\prime} \Psi^{\prime}, \quad H^{\prime}=\left(\begin{array}{cccc}
0 & g e^{\mathrm{i} \omega^{\prime} t} & 0 & -\mu B e^{\mathrm{i} \omega_{1} t} \\
g e^{-\mathrm{i} \omega^{\prime} t} & 0 & -\mu B e^{-\mathrm{i} \omega_{2} t} & 0 \\
0 & -\mu B e^{\mathrm{i} \omega_{2} t} & 0 & 0 \\
-\mu B e^{-\mathrm{i} \omega_{1} t} & 0 & 0 & 0
\end{array}\right),
$$

where [see also Eq. (2.8)]

$$
\begin{gathered}
\omega_{1}=E_{1}^{-}-E_{2}^{+}+\frac{g_{1}-g_{2}}{2} \approx 2 \Phi+g_{1}, \quad \omega_{2}=E_{1}^{+}-E_{2}^{-}+\frac{g_{1}-g_{2}}{2} \approx 2 \Phi-g_{2}, \\
\omega^{\prime}=E_{1}^{-}-E_{2}^{-}+\frac{g_{1}-g_{2}}{2} \approx 2 \Phi+2\left(g_{1}-g_{2}\right),
\end{gathered}
$$

and $\Phi=\delta m^{2} /(4 k)=\left(m_{1}^{2}-m_{2}^{2}\right) /(4 k)$ is the vacuum oscillation phase.

Let us next do the following transformation to the wave function:

$$
\begin{aligned}
& \Psi^{\prime}=\mathcal{U} \Psi \\
& \mathcal{U}=\operatorname{diag}\left\{e^{\mathrm{i}\left(\Phi+3 g_{1} / 4-g_{2} / 4\right) t}, e^{-\mathrm{i}\left(\Phi-3 g_{2} / 4+g_{1} / 4\right) t}, e^{\mathrm{i}\left(\Phi-g_{1} / 4-g_{2} / 4\right) t}, e^{-\mathrm{i}\left(\Phi+g_{1} / 4+g_{2} / 4\right) t}\right\} .
\end{aligned}
$$

This takes the Schrödinger Eq. (2.11) into the form

$$
\begin{aligned}
\mathrm{i} \frac{\mathrm{d} \Psi}{\mathrm{d} t} & =H \Psi, \quad H=\mathcal{U}^{\dagger} H^{\prime} \mathcal{U}-\mathrm{i} \mathcal{U}^{\dagger} \dot{\mathcal{U}}= \\
& =\left(\begin{array}{cccc}
\Phi+3 g_{1} / 4-g_{2} / 4 & g & 0 & -\mu B \\
g & -\Phi+3 g_{2} / 4-g_{1} / 4 & -\mu B & 0 \\
0 & -\mu B & \Phi-\left(g_{1}+g_{2}\right) / 4 & 0 \\
-\mu B & 0 & 0 & -\Phi-\left(g_{1}+g_{2}\right) / 4
\end{array}\right) .
\end{aligned}
$$

It is in order at this stage to compare the time evo- lution Eq. (2.13), which we obtained to in our approach 
based on Dirac equation, with the one obtained in the conventional quantum mechanical formalism. To this end let us introduce a quantum mechanical wave function $\Psi_{Q M}^{\mathrm{T}}=\left(\psi_{1}^{\mathrm{L}}, \psi_{2}^{\mathrm{L}}, \psi_{1}^{\mathrm{R}}, \psi_{2}^{\mathrm{R}}\right)$ for the neutrino mass eigenstates. Here $\psi_{a}^{\mathrm{L}, \mathrm{R}}$ are one-component objects. This wave function obeys the Schrödinger equation with the following effective Hamiltonian (see, e.g., Ref. [3]):

$$
H_{Q M}=\left(\begin{array}{cccc}
\mathcal{K}_{1}+g_{1} & g & 0 & -\mu B \\
g & \mathcal{K}_{2}+g_{2} & -\mu B & 0 \\
0 & -\mu B & \mathcal{K}_{1} & 0 \\
-\mu B & 0 & 0 & \mathcal{K}_{2}
\end{array}\right),
$$

where $\mathcal{K}_{a}=\sqrt{k^{2}+m_{a}^{2}} \approx k+m_{a}^{2} /(2 k)$ is the kinetic energy of a massive neutrino. It is easy to see that this matrix leads to the same dynamics as the Hamiltonian in Eq. (2.13). Indeed, without changing the dynamics one can make the following replacement $H_{Q M} \rightarrow H_{Q M}-$ $\operatorname{tr}\left(H_{Q M}\right) / 4 \cdot I$, where $I$ is the $4 \times 4$ unit matrix. The resulting Hamiltonian is exactly the same Hamiltonian we have derived in our approach.

To describe the evolution of the system (2.13) in a general case, one has to solve a secular equation which is the fourth-order algebraic equation (see also below). Although one can express the solution to such an equation in radicals, its actual form appears to be rather cumbersome for arbitrary parameters. If we, however, consider the case of a neutrino propagating in the electrically neutral isoscalar matter, i.e. $n_{e}=n_{p}$ and $n_{p}=n_{n}$, a reasonable solution is possible to find. We will demonstrate later that it corresponds to a realistic physical situation. As one can infer from Eq. (2.5) for the case of the $\nu_{e}^{\mathrm{L}} \rightarrow \nu_{\mu}^{\mathrm{R}}$ oscillations channel, in a medium with this property one has the effective potentials $f_{\alpha} \equiv f_{\mu}=$ $V_{\mu}=-G_{\mathrm{F}} n / \sqrt{2}$ and $f_{\beta} \equiv f_{e}=V_{e}=G_{\mathrm{F}} n / \sqrt{2}$, where $n \equiv n_{e}=n_{p}=n_{n}$. Using Eq. (2.4) we obtain that $g_{1}=-g_{2}=g_{0}$, where $g_{0}=-V \cos 2 \theta, g=V \sin 2 \theta$, and $V=G_{\mathrm{F}} n / \sqrt{2}$.

Let us point out that background matter with these properties may well exist in some astrophysical environments. The matter profile of presupernovae is poorly known, and a variety of presupernova models with different profiles exist in the literature (see, e.g. Ref. 21]). Nevertheless, electrically neutral isoscalar matter may well exist in the inner parts of presupernovae consisting of elements heavier than hydrogen. Indeed, for example, the model W02Z in Ref. 21] predicts that in a $15 M_{\odot}$ presupernova one has $Y_{e}=n_{e} /\left(n_{p}+n_{n}\right)=0.5$ in the $\mathrm{O}+\mathrm{Ne}+\mathrm{Mg}$ layer, between the $\mathrm{Si}+\mathrm{O}$ and He layers, in the radius range $(0.007-0.2) R_{\odot}$.

In this kind of background matter the effective Hamiltonian in Eq. (2.13) is replaced by

$$
H=\left(\begin{array}{cccc}
\Phi+g_{0} & g & 0 & -\mu B \\
g & -\left(\Phi+g_{0}\right) & -\mu B & 0 \\
0 & -\mu B & \Phi & 0 \\
-\mu B & 0 & 0 & -\Phi
\end{array}\right)
$$

We now look for the stationary solutions of the Schrödinger equation with this Hamiltonian. After a straightforward calculation one finds

$$
\begin{aligned}
\Psi(t)= & \sum_{\zeta= \pm 1}\left[\left(U_{\zeta} \otimes U_{\zeta}^{\dagger}\right) \exp \left(-\mathrm{i} \mathcal{E}_{\zeta} t\right)\right. \\
& \left.+\left(V_{\zeta} \otimes V_{\zeta}^{\dagger}\right) \exp \left(\mathrm{i} \mathcal{E}_{\zeta} t\right)\right] \Psi_{0},
\end{aligned}
$$

where we have denoted

$$
\begin{aligned}
\mathcal{E}_{ \pm} & =\frac{1}{2} \sqrt{2 V^{2}+4(\mu B)^{2}+4 \Phi^{2}-4 \Phi V \cos 2 \theta \pm 2 V R}, \\
R & =\sqrt{(V-2 \Phi \cos 2 \theta)^{2}+4(\mu B)^{2}} .
\end{aligned}
$$

The vectors $U_{ \pm}$and $V_{ \pm}$are the eigenvectors corresponding to the energy eigenvalues $\mathcal{E}_{ \pm}$and $-\mathcal{E}_{ \pm}$, respectively. They are given by $(\zeta= \pm)$

$$
\begin{gathered}
U_{\zeta}=\frac{1}{N_{\zeta}}\left(\begin{array}{c}
Z_{\zeta} \\
\sin 2 \theta\left(\mathcal{E}_{\zeta}-\Phi\right) \\
-\mu B \sin 2 \theta \\
-\mu B Z_{\zeta} /\left(\mathcal{E}_{\zeta}+\Phi\right)
\end{array}\right), \\
V_{\zeta}=\frac{1}{N_{\zeta}}\left(\begin{array}{c}
-\sin 2 \theta\left(\mathcal{E}_{\zeta}-\Phi\right) \\
Z_{\zeta} \\
\mu B Z_{\zeta} /\left(\mathcal{E}_{\zeta}+\Phi\right) \\
-\mu B \sin 2 \theta
\end{array}\right),
\end{gathered}
$$

where

$$
\begin{aligned}
Z_{\zeta}= & \frac{V+\zeta R}{2}-\mathcal{E}_{\zeta} \cos 2 \theta \\
N_{\zeta}^{2}= & Z_{\zeta}^{2}\left[1+\frac{(\mu B)^{2}}{\left(\mathcal{E}_{\zeta}+\Phi\right)^{2}}\right] \\
& +\sin ^{2}(2 \theta)\left[(\mu B)^{2}+\left(\mathcal{E}_{\zeta}-\Phi\right)^{2}\right] .
\end{aligned}
$$

It should be noted that Eq. (2.16) is the general solution of Eq. (2.13) satisfying the initial condition $\Psi(0)=\Psi_{0}$.

Note that we received the solution (2.16)-(2.19) of the evolution Eq. (2.13) under some assumptions on the external fields such as isoscalar matter with constant density and constant magnetic field. We mentioned above that our method is equivalent to the quantum mechanical description of neutrino oscillations [3] which is valid for a more general case of coordinate dependent external fields. The advantage of our approach consists in the fact that one can derive neutrinos' wave functions for arbitrary initial momenta, as it was made in Refs. [11, 12], and study the propagation of low-energy neutrinos. The assumption of constant matter density and magnetic field is quite realistic for certain astrophysical environments like a shock wave propagating inside an expanding envelope after a supernova explosion (see also Sec. V).

Consistently with Eqs. (2.1) and (2.2), we take the initial wave function $\Psi(0) \equiv \Psi_{0}$ in Eq. (2.16) as $\Psi_{0}^{\mathrm{T}}=$ $\left(\psi_{1}^{\mathrm{L}}, \psi_{2}^{\mathrm{L}}, \psi_{1}^{\mathrm{R}}, \psi_{2}^{\mathrm{R}}\right)=(\sin \theta, \cos \theta, 0,0)$. Using Eqs. (2.16)(2.19) one finds the components of the quantum mechanical wave function corresponding to the right-handed neu- 
trinos to be of the form

$$
\begin{aligned}
\psi_{1}^{\mathrm{R}}(t)= & \frac{\mu B}{N_{+}^{2}}\left\{\operatorname { c o s } \theta \left[e^{\mathrm{i} \mathcal{E}_{+} t} \frac{Z_{+}^{2}}{\mathcal{E}_{+}+\Phi}\right.\right. \\
& \left.-\sin ^{2}(2 \theta)\left(\mathcal{E}_{+}-\Phi\right) e^{-\mathrm{i} \mathcal{E}_{+} t}\right] \\
& \left.-\sin \theta \sin 2 \theta Z_{+}\left[e^{-\mathrm{i} \mathcal{E}_{+} t}+e^{\mathrm{i} \mathcal{E}_{+} t} \frac{\mathcal{E}_{+}-\Phi}{\mathcal{E}_{+}+\Phi}\right]\right\} \\
& +\{+\rightarrow-\}, \\
\psi_{2}^{\mathrm{R}}(t)= & \frac{\mu B}{N_{+}^{2}}\left\{\operatorname { s i n } \theta \left[\sin ^{2}(2 \theta)\left(\mathcal{E}_{+}-\Phi\right) e^{\mathrm{i} \mathcal{E}_{+} t}\right.\right. \\
& \left.-e^{-\mathrm{i} \mathcal{E}_{+} t} \frac{Z_{+}^{2}}{\mathcal{E}_{+}+\Phi}\right] \\
& \left.-\cos \theta \sin 2 \theta Z_{+}\left[e^{\mathrm{i} \mathcal{E}_{+} t}+e^{-\mathrm{i} \mathcal{E}_{+} t} \frac{\mathcal{E}_{+}-\Phi}{\mathcal{E}_{+}+\Phi}\right]\right\} \\
& +\{+\rightarrow-\},
\end{aligned}
$$

where the $\{+\rightarrow-\}$ stand for the terms similar to the terms preceding each of them but with all quantities with a subscript + replaced with corresponding quantities with a subscript - . The wave function of the right-handed neutrino of the flavor $\alpha, \nu_{\alpha}^{\mathrm{R}}$, can be written with help of Eqs. (2.1) and (2.20) as $\nu_{\alpha}^{\mathrm{R}}(t)=$ $\cos \theta \psi_{1}^{\mathrm{R}}(t)-\sin \theta \psi_{2}^{\mathrm{R}}(t)$.

The probability for the transition $\nu_{\beta}^{\mathrm{L}} \rightarrow \nu_{\alpha}^{\mathrm{R}}$ is obtained as the square of the quantum mechanical wave function $\nu_{\alpha}^{\mathrm{R}}$. One obtains

$$
\begin{aligned}
P_{\nu_{\beta}^{\mathrm{L}} \rightarrow \nu_{\alpha}^{\mathrm{R}}}(t)= & \left|\nu_{\alpha}^{\mathrm{R}}\right|^{2}=\left[C_{+} \cos \left(\mathcal{E}_{+} t\right)+C_{-} \cos \left(\mathcal{E}_{-} t\right)\right]^{2} \\
& +\left[S_{+} \sin \left(\mathcal{E}_{+} t\right)+S_{-} \sin \left(\mathcal{E}_{-} t\right)\right]^{2}
\end{aligned}
$$

where $(\zeta= \pm)$

$$
\begin{aligned}
C_{\zeta}= & \frac{\mu B}{N_{\zeta}^{2}}\left\{\frac{Z_{\zeta}^{2}}{\mathcal{E}_{\zeta}+\Phi}-\sin ^{2}(2 \theta)\left(\mathcal{E}_{\zeta}-\Phi\right)\right\}, \\
S_{\zeta}= & \frac{\mu B}{N_{\zeta}^{2}}\left\{\sin ^{2}(2 \theta) \frac{2 \Phi Z_{\zeta}}{\mathcal{E}_{\zeta}+\Phi}\right. \\
& \left.+\cos 2 \theta\left[\frac{Z_{\zeta}^{2}}{\mathcal{E}_{\zeta}+\Phi}+\sin ^{2}(2 \theta)\left(\mathcal{E}_{\zeta}-\Phi\right)\right]\right\} .
\end{aligned}
$$

As a consistency check, one easily finds from Eq. (2.22) that $C_{+}+C_{-}=0$ as required for assuring $P(0)=0$.
In the following we will limit our considerations to the case $\mathcal{E}_{+} \approx \mathcal{E}_{-}$, corresponding to the situations where the effect of the interactions of neutrinos with matter $(V)$ is small compared with that of the magnetic interactions $(\mu B)$ or the vacuum contribution $(\Phi)$ or both [see Eq. (2.17)]. Note that in this case one can analyze the exact oscillation probability (2.21) analytically, which would be practically impossible in more general situations.

In the case $\mathcal{E}_{+} \approx \mathcal{E}_{-}$, one can present the transition probability in Eq. (2.21) in the following form:

$$
P(t)=P_{0}(t)+P_{c}(t) \cos (2 \Omega t)+P_{s}(t) \sin (2 \Omega t),
$$
where

$$
\begin{aligned}
P_{0}(t)= & \frac{1}{2}\left[S_{+}^{2}+S_{-}^{2}+2 S_{+} S_{-} \cos (2 \delta \Omega t)\right. \\
& \left.-4 C_{+} C_{-} \sin ^{2}(\delta \Omega t)\right], \\
P_{c}(t)= & -\frac{1}{2}\left[\left(S_{+}^{2}+S_{-}^{2}\right) \cos (2 \delta \Omega t)+2 S_{+} S_{-}\right. \\
& \left.-4 C_{+} C_{-} \sin ^{2}(\delta \Omega t)\right], \\
P_{s}(t)= & \frac{1}{2}\left(S_{+}^{2}-S_{-}^{2}\right) \sin (2 \delta \Omega t),
\end{aligned}
$$

and

$$
\Omega=\frac{\mathcal{E}_{+}+\mathcal{E}_{-}}{2}, \quad \delta \Omega=\frac{\mathcal{E}_{+}-\mathcal{E}_{-}}{2}
$$

As one can infer from these expressions, the transition probability $P(t)$ is a rapidly oscillating function, with the frequency $\Omega$, enveloped from up and down by the slowly varying functions $P_{u, d}=P_{0} \pm \sqrt{P_{c}^{2}+P_{s}^{2}}$, respectively.

The behavior of the transition probability for various matter densities $\rho$ and the values of $\mu B$ and for a fixed neutrino energy of $E=10 \mathrm{MeV}$ and squared mass difference of $\delta m^{2}=8 \times 10^{-5} \mathrm{eV}^{2}$ is illustrated in Figs. 1] 3 .

As these plots show, at low matter densities the envelope functions give, at each propagation distance, the range of the possible values of the oscillation probability. At greater matter densities, where the probability oscillates less intensively, the envelope functions are not that useful in analyzing the physical situation.

One can find the maximum value of the upper envelope function, which is also the upper bound for the transition probability, given as

$$
P_{u}^{(\max )}= \begin{cases}\left(S_{+}-S_{-}\right)^{2}, & \text { if } B<B^{\prime}, \\ C_{+} C_{-}\left(S_{+}^{2}-S_{-}^{2}\right)^{2}\left[C_{+} C_{-}\left(S_{+}^{2}+S_{-}^{2}\right)+\left(C_{+} C_{-}\right)^{2}+\left(S_{+} S_{-}\right)^{2}\right]^{-1}, & \text { if } B>B^{\prime},\end{cases}
$$

where the value $B^{\prime}$ is the solution of the transcendent algebraic equation, $C_{+} C_{-}=S_{+} S_{-}$. The corresponding maximum values of the averaged transition probablility 


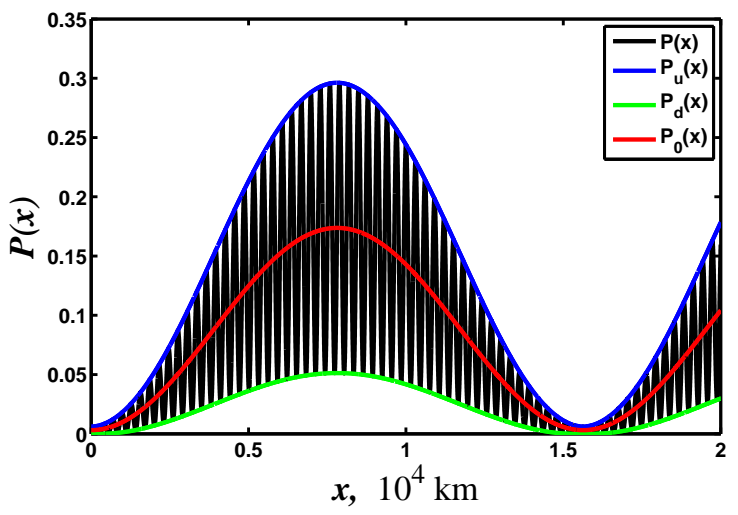

(a)

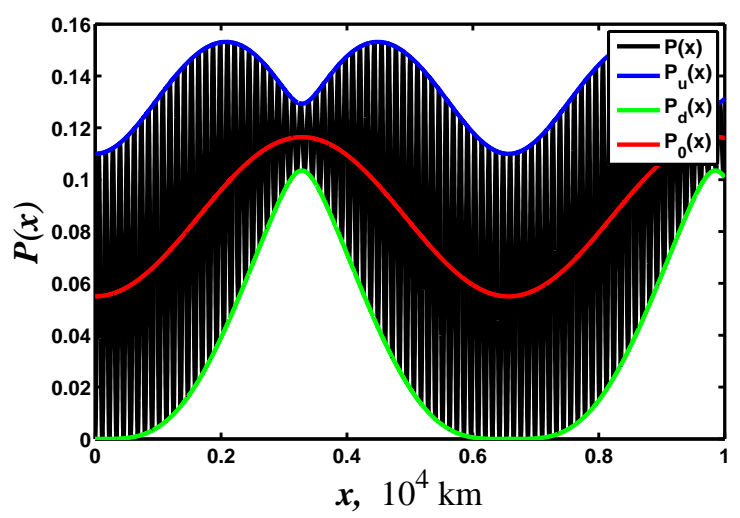

(c)

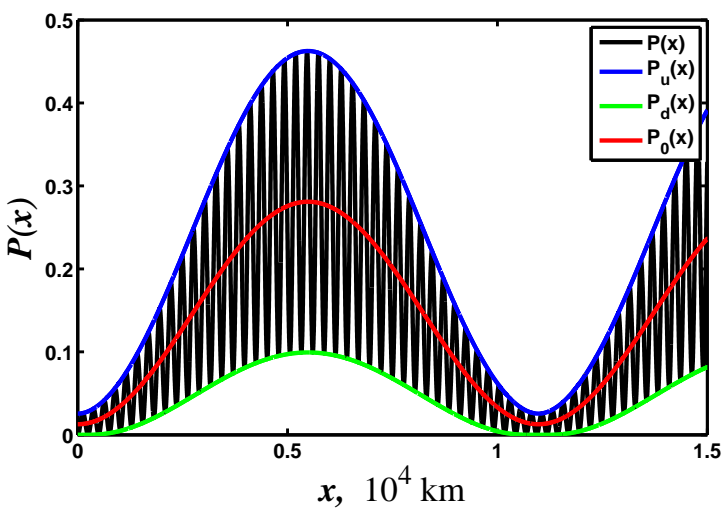

(b)

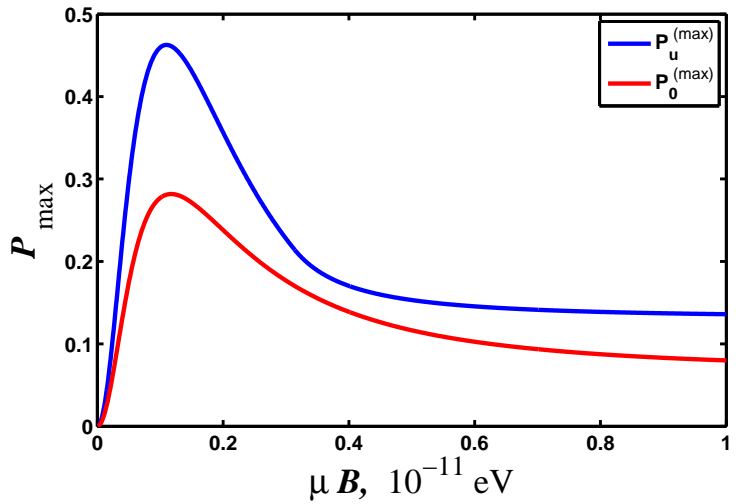

(d)

FIG. 1: (a)-(c) The transition probability versus the distance passed by a neutrino beam in matter with the density $\rho=10 \mathrm{~g} / \mathrm{cc}$; (a) $\mu B=5 \times 10^{-13} \mathrm{eV}$, (b) $\mu B=1.1 \times 10^{-12} \mathrm{eV}$, (c) $\mu B=5 \times 10^{-12} \mathrm{eV}$. We take that $E_{\nu}=10 \mathrm{MeV}^{2} \delta m^{2}=8 \times 10^{-5} \mathrm{eV}^{2}$ and $\theta=0.6$, which is quite close to the solar neutrinos' oscillations parameters. The black line is the function $P(x)$, the blue and green lines are the envelope functions $P_{u, d}(x)$, and the red line is the averaged transition probability $P_{0}(x)$. (d) The dependence of the maximal values of the functions $P(x)$ and $P_{0}(x)$, blue and red lines, respectively, on the magnetic energy $\mu B$ for the given density.

$P_{0}(x)$ are given by

$$
P_{0}^{(\max )}=\frac{1}{2}\left[\left(S_{+} S_{-}\right)^{2}-4 C_{+} C_{-}\right],
$$

for arbitrary values of $B$. The values of these maxima depend on the size of the quantity $\mu B$. These dependencies are plotted in Figs. 1(d)-3(d). In the case of rapid oscillations the physically relevant quantities, rather than the maxima, are the averaged values of the transition probability, which are also plotted in these figures.

As Figs. 1(d)-3(d) show, the interplay of the matter effect and the magnetic interaction can lead, for a given magnetic moment $\mu$, to an enhanced spin-flavor transition if the magnetic field $B$ has a suitable strength relative to the density of matter $\rho$. In our numerical examples this occurs at $\mu B_{\max }=1.1 \times 10^{-12} \mathrm{eV}$ for $\rho=10 \mathrm{~g} / \mathrm{cc}$, at $\mu B_{\max }=6.6 \times 10^{-13} \mathrm{eV}$ for $\rho=50 \mathrm{~g} / \mathrm{cc}$, and at $\mu B_{\max }=8 \times 10^{-13} \mathrm{eV}$ for $\rho=100 \mathrm{~g} / \mathrm{cc}$. For these values of $\mu B$ both the maxima and the average of the transition probability become considerably larger than for any other values of $\mu B$. Figures 1 (b)-33 (b) correspond to the situation of maximal enhancement, whereas Figs. 1(a)3(a) and Figs.11(c)-3(c) illustrate the situation above and below the optimal strength $B_{\max }$ of the magnetic field.

It is noteworthy that the enhanced transition probability is achieved towards the lower end of the $\mu B$ region where substantial transitions all occur, that is, at relatively moderate magnetic fields. At larger values of $\mu B$ the maximum of the transition probability approaches towards $\cos ^{2}(2 \theta)$. Indeed, if $\mu B \gg \max (\Phi, V)$, the transition probability can be written in the form (see Ref. [13]) $P(t)=\cos ^{2}(2 \theta) \sin ^{2}(\mu B t)$. It was found in Ref. 22 that neutrino spin-flavor oscillations can be enhanced in a very strong magnetic field, with the transition probability being practically equal to unity. This phenomenon can be realized only for Dirac neutrinos with small off-diagonal magnetic moments and small mixing angle. As we can see from Figs. 1(d)-3(d) the situation is completely different for big off-diagonal magnetic moments.

One should notice that for long propagation distances 


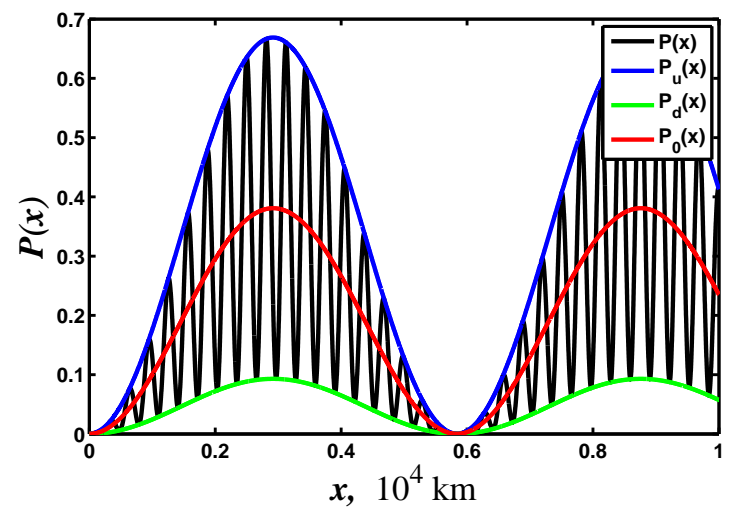

(a)

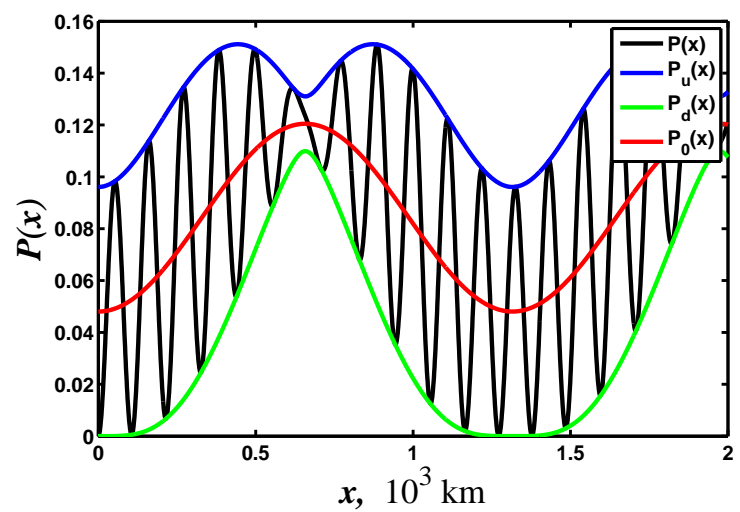

(c)

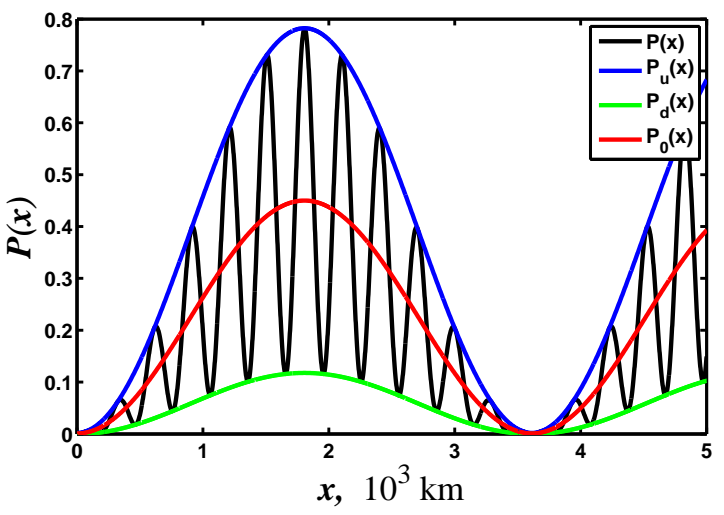

(b)

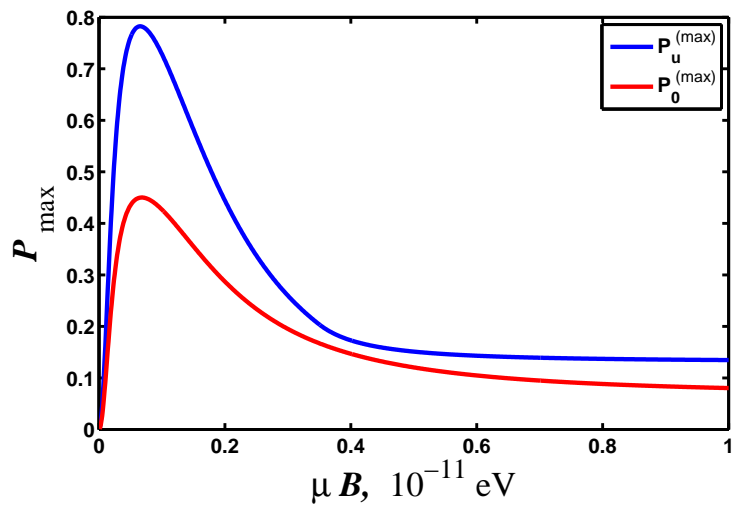

(d)

FIG. 2: The same as in Fig. 1 for the density $\rho=50 \mathrm{~g} / \mathrm{cc}$; (a) $\mu B=3.5 \times 10^{-13} \mathrm{eV}$, (b) $\mu B=6.6 \times 10^{-13} \mathrm{eV},(\mathrm{c}) \mu B=$ $5 \times 10^{-12} \mathrm{eV}$.

consisting of several oscillation periods of the envelope functions, the enhancement effect would diminish considerably due to averaging. In the numerical examples presented in Figs. 1] 3 the period of the envelope function is of the order of $10^{3}-10^{4} \mathrm{~km}$, which is a typical size of a shock wave with the matter densities we have used in the plots (see, e.g., Ref. [23]). Thus the enhanced spinflavor transition could take place when neutrinos traverse a shock wave.

Let us recall that the above analysis was made by assuming neutrinos to be Dirac particles. We will see below (see Sec. IV) that the corresponding results are quite different in the case of Majorana neutrinos.

\section{EVOLUTION OF MAJORANA NEUTRINOS IN VACUUM}

We now move to consider Majorana neutrinos, and we shall start by applying our formalism to the ordinary vacuum oscillation of two Majorana neutrinos. The left-handed chirality component of a flavor neutrino $\nu_{\lambda}^{\mathrm{L}}=(1 / 2)\left(1-\gamma^{5}\right) \nu_{\lambda}$ is related to the wave functions of
Majorana neutrino states through

$$
\nu_{\lambda}^{\mathrm{L}}=\sum_{a} U_{\lambda a} \eta_{a}
$$

where $\lambda=\alpha, \beta$ is the flavor index and $\eta_{a}, a=1,2$, correspond to a Majorana particle with a definite mass $m_{a}$. In the simplest case the mixing of the flavor states arises purely from Majorana mass terms between the lefthanded neutrinos, and then the mixing matrix $U_{\lambda a}$ is a $2 \times 2$ and unitary matrix, i.e., $a=1,2$ and, assuming no $\mathrm{CP}$ violation, it can be parametrized in the same way as in Eq. (2.1).

We study the evolution of this system with the following initial condition [see also Eq. (2.2)]:

$$
\nu_{\alpha}^{\mathrm{L}}(\mathbf{r}, 0)=0, \quad \nu_{\beta}^{\mathrm{L}}(\mathbf{r}, 0)=\nu_{\beta}^{(0)} e^{\mathrm{ikr}},
$$

where $\mathbf{k}=(0,0, k)$ is the initial momentum and $\nu_{\beta}^{(0) \mathrm{T}}=$ $(0,1)$. The initial state is thus a left-handed neutrino of flavor $\beta$ propagating along the $z$-axis to the positive direction.

As both the left-handed state $\nu_{\lambda}^{\mathrm{L}}$ and Majorana state $\eta_{a}$ have two degrees of freedom, we will describe them in 


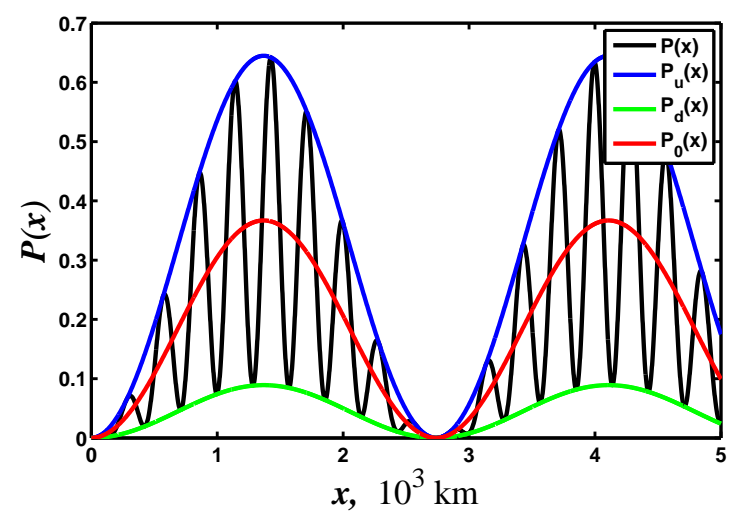

(a)

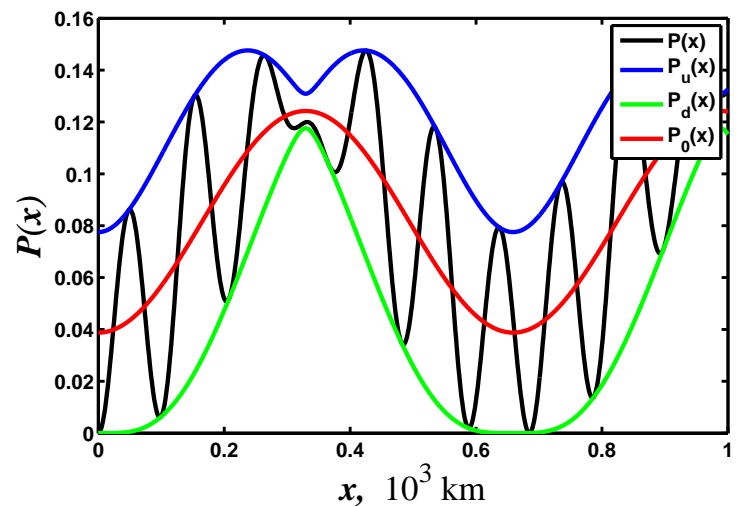

(c)

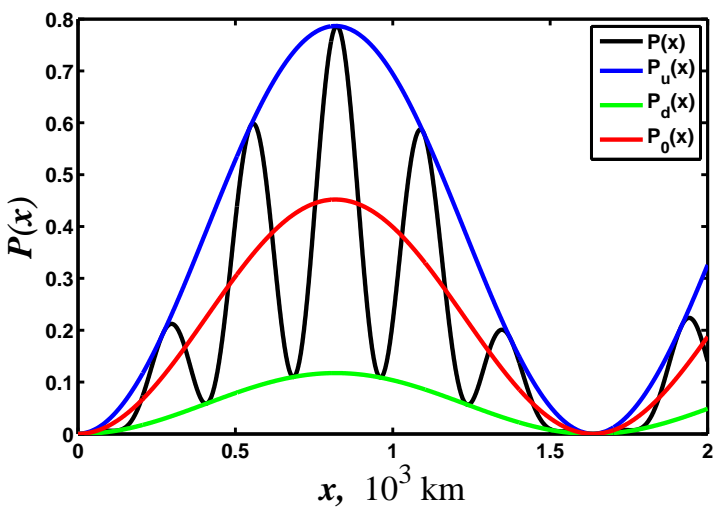

(b)

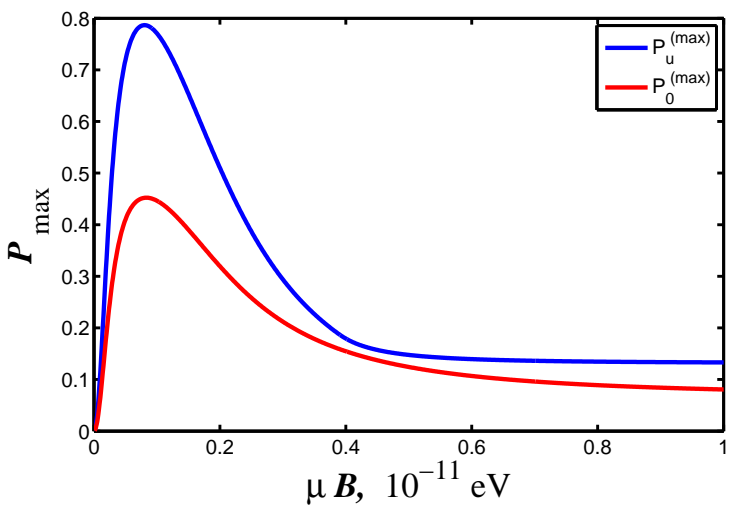

(d)

FIG. 3: The same as in Fig. 1 for the density $\rho=100 \mathrm{~g} / \mathrm{cc}$; (a) $\mu B=4 \times 10^{-13} \mathrm{eV}$, (b) $\mu B=8 \times 10^{-13} \mathrm{eV},(\mathrm{c}) \mu B=5 \times 10^{-12} \mathrm{eV}$.

the following by using two-component Weyl spinors. The Weyl spinor of a free Majorana particle obeys the wave equation (see, e.g., [24]),

$$
\mathrm{i} \dot{\eta}_{a}+(\boldsymbol{\sigma} \mathbf{p}) \eta_{a}+\mathrm{i} m_{a} \sigma_{2} \eta_{a}^{*}=0 .
$$

The general solution of this equation can be presented as [25]

$$
\begin{aligned}
\eta_{a}(\mathbf{r}, t)= & \int \frac{\mathrm{d}^{3} \mathbf{p}}{(2 \pi)^{3 / 2}} e^{\mathrm{i} \mathbf{p r}} \sum_{\zeta= \pm 1}\left[a_{a}^{(\zeta)}(\mathbf{p}) u_{a}^{(\zeta)}(\mathbf{p}) e^{-\mathrm{i} E_{a} t}\right. \\
& \left.+a_{a}^{(\zeta) *}(-\mathbf{p}) v_{a}^{(\zeta)}(-\mathbf{p}) e^{\mathrm{i} E_{a} t}\right]
\end{aligned}
$$

where $E_{a}=\sqrt{m_{a}^{2}+|\mathbf{p}|^{2}}$. The basis spinors $u_{a}^{(\zeta)}$ and $v_{a}^{(\zeta)}$ have the form

$$
\begin{array}{ll}
u_{a}^{+}(\mathbf{p})=-\lambda_{a} \frac{m_{a}}{E_{a}+|\mathbf{p}|} w_{+}, & u_{a}^{-}(\mathbf{p})=\lambda_{a} w_{-}, \\
v_{a}^{+}(\mathbf{p})=\lambda_{a} w_{-}, & v_{a}^{-}(\mathbf{p})=\lambda_{a} \frac{m_{a}}{E_{a}+|\mathbf{p}|} w_{+},
\end{array}
$$

where $w_{ \pm}$are helicity amplitudes given by 26 ]

$$
\begin{aligned}
& w_{+}=\left(\begin{array}{c}
e^{-i \phi / 2} \cos (\vartheta / 2) \\
e^{i \phi / 2} \sin (\vartheta / 2)
\end{array}\right), \\
& w_{-}=\left(\begin{array}{c}
-e^{-i \phi / 2} \sin (\vartheta / 2) \\
e^{i \phi / 2} \cos (\vartheta / 2)
\end{array}\right),
\end{aligned}
$$

the angles $\phi$ and $\vartheta$ giving the direction of the momentum of the particle, $\mathbf{p}=|\mathbf{p}|(\sin \vartheta \cos \phi, \sin \vartheta \sin \phi, \cos \vartheta)$. The normalization factor $\lambda_{a}$ in Eq. (3.5) can be chosen as

$$
\lambda_{a}^{-2}=1-\frac{m_{a}^{2}}{\left(E_{a}+|\mathbf{p}|\right)^{2}} .
$$

Let us mention the following properties of the helicity amplitudes $w_{ \pm}$:

$$
\begin{gathered}
(\boldsymbol{\sigma} \mathbf{p}) w_{ \pm}= \pm|\mathbf{p}| w_{ \pm}, \quad \mathrm{i} \sigma_{2} w_{ \pm}^{*}=\mp w_{\mp}, \\
w_{ \pm}(-\mathbf{p})=\mathrm{i} w_{\mp}(\mathbf{p}), \\
\left(w_{+} \otimes w_{-}^{\mathrm{T}}\right)-\left(w_{-} \otimes w_{+}^{\mathrm{T}}\right)=\mathrm{i} \sigma_{2}, \\
\left(w_{+} \otimes w_{+}^{\dagger}\right)+\left(w_{-} \otimes w_{-}^{\dagger}\right)=1,
\end{gathered}
$$

which can be immediately obtained from Eq. (3.6) and which are useful in deriving the results given below.

The time-independent coefficients $a_{a}^{ \pm}(\mathbf{p})$ in Eq. (3.4) 
have the following form [25]:

$$
\begin{aligned}
a_{a}^{+}(\mathbf{p})= & \frac{1}{(2 \pi)^{3 / 2}}\left[\eta_{a}^{(0) \dagger}(-\mathbf{p}) v_{a}^{+}(\mathbf{p})\right. \\
& \left.+\frac{\mathrm{i} m_{a}}{E_{a}+|\mathbf{p}|} v_{a}^{+\dagger}(-\mathbf{p}) \eta_{a}^{(0)}(\mathbf{p})\right], \\
a_{a}^{-}(\mathbf{p})= & \frac{1}{(2 \pi)^{3 / 2}}\left[u_{a}^{-\dagger}(\mathbf{p}) \eta_{a}^{(0)}(\mathbf{p})\right. \\
& \left.-\frac{\mathrm{i} m_{a}}{E_{a}+|\mathbf{p}|} \eta_{a}^{(0) \dagger}(-\mathbf{p}) u_{a}^{-}(-\mathbf{p})\right],
\end{aligned}
$$

where $\eta_{a}^{(0)}(\mathbf{p})$ is the Fourier transform of the initial wave function $\eta_{a}$,

$$
\eta_{a}^{(0)}(\mathbf{p})=\int \mathrm{d}^{3} \mathbf{p} e^{-\mathrm{ipr}} \eta_{a}^{(0)}(\mathbf{r}) .
$$

Using Eqs. (3.4)-(3.9) we then obtain the following expression for the wave function for the neutrino mass eigenstates:

$$
\begin{aligned}
\eta_{a}(\mathbf{r}, t)= & \int \frac{\mathrm{d}^{3} \mathbf{p}}{(2 \pi)^{3}} e^{\mathrm{i} \mathbf{p r}} \lambda_{a}^{2} \\
& \times\left[\left\{\left(e^{-\mathrm{i} E_{a} t}-\left[\frac{m_{a}}{E_{a}+|\mathbf{p}|}\right]^{2} e^{\mathrm{i} E_{a} t}\right)\right.\right. \\
& \times\left(w_{-} \otimes w_{-}^{\dagger}\right) \\
& +\left(e^{\mathrm{i} E_{a} t}-\left[\frac{m_{a}}{E_{a}+|\mathbf{p}|}\right]^{2} e^{-\mathrm{i} E_{a} t}\right) \\
& \left.\times\left(w_{+} \otimes w_{+}^{\dagger}\right)\right\} \eta_{a}^{(0)}(\mathbf{p}) \\
& \left.-2 \frac{m_{a}}{E_{a}+|\mathbf{p}|} \sin \left(E_{a} t\right) \sigma_{2} \eta_{a}^{(0) *}(-\mathbf{p})\right] .
\end{aligned}
$$

From Eqs. (3.8) and (3.10) it follows that a mass eigenstate particle initially in the left-polarized state $\eta_{a}^{(0)}(\mathbf{r}) \sim w_{-}(\mathbf{k}) e^{\mathrm{ikr}}$ is described at later times by

$$
\begin{aligned}
\eta_{a}(\mathbf{r}, t) \sim \lambda_{a}^{2} & \left\{\left(e^{-\mathrm{i} E_{a} t}-\left[\frac{m_{a}}{E_{a}+|\mathbf{k}|}\right]^{2} e^{\mathrm{i} E_{a} t}\right) e^{\mathrm{i} \mathbf{k r}} w_{-}(\mathbf{k})\right. \\
& \left.-2 \mathrm{i} \frac{m_{a}}{E_{a}+|\mathbf{k}|} \sin \left(E_{a} t\right) e^{-\mathrm{i} \mathbf{k r}} w_{+}(\mathbf{k})\right\}
\end{aligned}
$$

Let us notice that the second term in Eq. (3.11) describes an antineutrino state. Indeed the spinor $w_{+}(\mathbf{k})$ satisfies the relation, $(\boldsymbol{\sigma} \mathbf{k}) w_{+}(\mathbf{k})=|\mathbf{k}| w_{+}(\mathbf{k})$, see Eq. (3.8). Therefore it corresponds to an antiparticle, see Ref. [27]. This term is responsible for the neutrino-to-antineutrino flavor state transition $\nu_{\beta}^{\mathrm{L}} \leftrightarrow\left(\nu_{\alpha}^{\mathrm{L}}\right)^{c}$.

According to Eq. (3.1) and (2.1), the wave function of the left-handed neutrino of flavor $\alpha$ is $\nu_{\alpha}^{\mathrm{L}}=\cos \theta \eta_{1}^{\mathrm{L}}-$ $\sin \theta \eta_{2}^{\mathrm{L}}$. From Eqs. (3.1) and (3.11) it then follows that the probability of the transition $\nu_{\beta}^{\mathrm{L}} \rightarrow \nu_{\alpha}^{\mathrm{L}}$ in vacuum is given by

$$
\begin{aligned}
P_{\nu_{\beta}^{\mathrm{L}} \rightarrow \nu_{\alpha}^{\mathrm{L}}}(t)= & \left|\nu_{\alpha}^{\mathrm{L}}\right|^{2}=\sin ^{2}(2 \theta)\left\{\sin ^{2}(\Phi t)\right. \\
& +\frac{1}{4|\mathbf{k}|^{2}} \cos (|\mathbf{k}| t) \sin (\Phi t) \\
& \left.\times\left[m_{1}^{2} \sin \left(E_{1} t\right)-m_{2}^{2} \sin \left(E_{2} t\right)\right]\right\} \\
& +\mathcal{O}\left(\frac{m_{a}}{|\mathbf{k}|}\right)^{4} .
\end{aligned}
$$

The leading term reproduces the familiar oscillation formula of Pontecorvo describing the transitions between active neutrinos $\nu_{\beta}^{\mathrm{L}} \leftrightarrow \nu_{\alpha}^{\mathrm{L}}$. The corrections to Pontecorvo's formula were obtained first in Ref. [28], and in our previous papers 11, 12, 13, 14] we derived the analogous corrections for Dirac neutrinos both in vacuum and in various external fields.

Analogously we can calculate the transition probability for the process $\nu_{\beta}^{\mathrm{L}} \rightarrow\left(\nu_{\alpha}^{\mathrm{L}}\right)^{c}$ using the second term in Eq. (3.11),

$$
\begin{aligned}
P_{\nu_{\beta}^{\mathrm{L}} \rightarrow\left(\nu_{\alpha}^{\mathrm{L}}\right)^{c}}(t)= & \left|\left(\nu_{\alpha}^{\mathrm{L}}\right)^{c}\right|^{2}=\frac{\sin ^{2}(2 \theta)}{4|\mathbf{k}|^{2}} \\
& \times\left[m_{1} \sin \left(E_{1} t\right)-m_{2} \sin \left(E_{2} t\right)\right]^{2} \\
& +\mathcal{O}\left(\frac{m_{a}}{|\mathbf{k}|}\right)^{4} .
\end{aligned}
$$

Note that the next-to-leading term in Eq. (3.12) and leading term in Eq. (3.13) have the same order of magnitude $\sim m_{a}^{2} /|\mathbf{k}|^{2}$.

Before moving to consider Majorana neutrinos in magnetic fields we make a general comment concerning the validity of our approach based on relativistic classical field theory. It has been stated [2] that the dynamics of massive Majorana fields cannot be described within the classical field theory approach due to the fact that the mass term of the Lagrangian, $\eta^{\mathrm{T}} \mathrm{i} \sigma_{2} \eta$, vanishes when $\eta$ is represented as a $c$-number function. Note that Eq. (3.3) is a direct consequence of the Dirac equation if we suggest that the four-component wave function satisfies the Majorana condition. Therefore a solution to Eq. (3.3), i.e., wave functions and energy levels, in principle does not depend on the existence of a Lagrangian resulting in this equation. The wave equations describing elementary particles should follow from the quantum field theory principles. However quite often these quantum equations allow classical solutions (see Ref. [29] for many interesting examples). We have also demonstrated in Refs. 11, 12, 13, 14] that oscillations of Dirac neutrinos in vacuum and various external fields can be described in the framework of the classical field theory. The main result of this section was to show that the quantum Eq. (3.3) for massive Majorana particles can be solved [see Eq. (3.11)] in the framework of the classical field theory as well. 


\section{EVOLUTION OF MAJORANA NEUTRINOS IN MATTER AND TRANSVERSAL MAGNETIC FIELD}

For describing the evolution of two Majorana mass eigenstates in matter under the influence of an external magnetic field, the wave Eq. (3.3) is to be modified to the following form:

$$
\begin{aligned}
\mathrm{i} \dot{\eta}_{a}+\left(\boldsymbol{\sigma} \mathbf{p}-\frac{g_{a}}{2}\right) & \eta_{a}+\mathrm{i} m_{a} \sigma_{2} \eta_{a}^{*}-\frac{g}{2} \eta_{b} \\
& -\mathrm{i} \mu(\boldsymbol{\sigma} \mathbf{B}) \sigma_{2} \epsilon_{a b} \eta_{b}^{*}=0, \quad a \neq b,
\end{aligned}
$$

where $\epsilon_{a b}=\mathrm{i}\left(\sigma_{2}\right)_{a b}$, and $g_{a}$ and $g$ were defined in connection to Eq. (2.3). Note that Eq. (4.1) can be formally derived from Eq. (2.3) if one neglects vector current interactions, i.e., replace $\left(1-\gamma^{5}\right) / 2$ with $-\gamma^{5} / 2$, and takes into account the fact that the magnetic moment matrix of Majorana neutrinos is antisymmetric (see, e.g., Ref. [30]). We will apply the same initial condition (3.2) as in the vacuum case. It should be mentioned that the evolution of Majorana neutrinos in matter and in a magnetic field has been previously discussed in Ref. [31].

The general solution of Eq. (4.1) can be expressed in the following form:

$$
\begin{aligned}
\eta_{a}(\mathbf{r}, t)= & \int \frac{\mathrm{d}^{3} \mathbf{p}}{(2 \pi)^{3 / 2}} e^{\mathrm{i} \mathbf{p r}} \\
& \times \sum_{\zeta= \pm 1}\left[a_{a}^{(\zeta)}(\mathbf{p}, t) u_{a}^{(\zeta)}(\mathbf{p}) \exp \left(-\mathrm{i} E_{a}^{(\zeta)} t\right)\right. \\
& \left.+a_{a}^{(\zeta) *}(-\mathbf{p}, t) v_{a}^{(\zeta)}(-\mathbf{p}) \exp \left(\mathrm{i} E_{a}^{(\zeta)} t\right)\right]
\end{aligned}
$$

where the energy levels are given in Eq. 2.7) (see Ref. 20]). The basis spinors in Eq. (4.2) can be chosen as

$$
\begin{aligned}
& u_{a}^{+}(\mathbf{p})=-\lambda_{a}^{+} \frac{m_{a}}{E_{a}^{+}+\left(|\mathbf{p}|-g_{a} / 2\right)} w_{+}, \\
& v_{a}^{-}(\mathbf{p})=\lambda_{a}^{-} \frac{m_{a}}{E_{a}^{-}+\left(|\mathbf{p}|+g_{a} / 2\right)} w_{+}, \\
& u_{a}^{-}(\mathbf{p})=\lambda_{a}^{-} w_{-}, \quad v_{a}^{+}(\mathbf{p})=\lambda_{a}^{+} w_{-},
\end{aligned}
$$

where the normalization factors $\lambda_{a}^{(\zeta)}, \zeta= \pm$ are given by

$$
\left(\lambda_{a}^{(\zeta)}\right)^{-2}=1-\frac{m_{a}^{2}}{\left[E_{a}+\left(|\mathbf{p}|-\zeta g_{a} / 2\right)\right]^{2}} .
$$

Let us consider the propagation of Majorana neutrinos in the transversal magnetic field. Using a similar technique as in the Dirac case in Sec. III and assuming $k \gg m_{a}$, we end up with the following ordinary differential equations for the coefficients $a_{a}^{(\zeta)}$,

$\mathrm{i} \frac{\mathrm{d} \Psi^{\prime}}{\mathrm{d} t}=H^{\prime} \Psi^{\prime}$

$$
H^{\prime}=\left(\begin{array}{cccc}
0 & g e^{\mathrm{i} \delta_{-} t} / 2 & 0 & \mu B e^{\mathrm{i} \sigma_{+} t} \\
g e^{-\mathrm{i} \delta_{-} t} / 2 & 0 & -\mu B e^{-\mathrm{i} \sigma_{-} t} & 0 \\
0 & -\mu B e^{\mathrm{i} \sigma_{-} t} & 0 & -g e^{\mathrm{i} \delta_{+} t} / 2 \\
\mu B e^{-\mathrm{i} \sigma_{+} t} & 0 & -g e^{-\mathrm{i} \delta_{+} t} / 2 & 0
\end{array}\right)
$$
where $\Psi^{\prime T}=\left(a_{1}^{-}, a_{2}^{-}, a_{1}^{+}, a_{2}^{+}\right)$and

$$
\begin{aligned}
& \delta_{ \pm}=E_{1}^{ \pm}-E_{2}^{ \pm} \approx 2 \Phi \mp \frac{g_{1}-g_{2}}{2}, \\
& \sigma_{ \pm}=E_{1}^{\mp}-E_{2}^{ \pm} \approx 2 \Phi \pm \frac{g_{1}+g_{2}}{2} .
\end{aligned}
$$

By making the matrix transformation

$$
\Psi^{\prime}=\mathcal{U} \Psi, \quad \mathcal{U}=\operatorname{diag}\left\{e^{\mathrm{i}\left(\Phi+g_{1} / 2\right) t}, e^{-\mathrm{i}\left(\Phi-g_{2} / 2\right) t}, e^{\mathrm{i}\left(\Phi-g_{1} / 2\right) t}, e^{-\mathrm{i}\left(\Phi+g_{2} / 2\right) t}\right\},
$$

we can recast Eq. (4.3) into the form

$$
\mathrm{i} \frac{\mathrm{d} \Psi}{\mathrm{d} t}=H \Psi, \quad H=\mathcal{U}^{\dagger} H^{\prime} \mathcal{U}-\mathrm{i} \mathcal{U}^{\dagger} \dot{\mathcal{U}}=\left(\begin{array}{cccc}
\Phi+g_{1} / 2 & g / 2 & 0 & \mu B \\
g / 2 & -\Phi+g_{2} / 2 & -\mu B & 0 \\
0 & -\mu B & \Phi-g_{1} / 2 & -g / 2 \\
\mu B & 0 & -g / 2 & -\Phi-g_{2} / 2
\end{array}\right) .
$$

Let us note that the analogous effective Hamiltonian has been used in describing the spin-flavor oscillations of Majorana neutrinos within the quantum mechanical approach (see, e.g., Ref. [3]) if we use the basis $\Psi_{Q M}^{\mathrm{T}}=\left(\psi_{1}^{\mathrm{L}}, \psi_{2}^{\mathrm{L}},\left[\psi_{1}^{\mathrm{L}}\right]^{c},\left[\psi_{2}^{\mathrm{L}}\right]^{c}\right)$.

Note that the consistent derivation of the master Eq. (4.1) should be done in the framework of the quantum field theory (see, e.g., Ref. [2]), supposing that the spinors $\eta_{a}$ are expressed via anticommuting operators. This quantum field theory treatment is important to explain the asymmetry of the magnetic moment matrix. However, it is possible to see that the main Eq. (4.1) can also be reduced to the standard Schrödinger evolution Eq. (4.5) for neutrino spin-flavor oscillations if we suppose that the wave functions $\eta_{a}$ are $c$-number objects. That is why one can again conclude that classical 
and quantum field theory methods for studying Majorana neutrinos' propagation in external fields are equivalent.

Let us again consider the situation when $n_{e}=n_{p}=$ $n_{n}=n$, which results in $g_{1}=-g_{2}$. In this case the eigenvalues of the Hamiltonian (4.5) $\lambda= \pm \mathcal{E}_{ \pm}$are given by

$$
\begin{aligned}
\mathcal{E}_{ \pm} & =\frac{1}{2} \sqrt{V^{2}+4(\mu B)^{2}+4 \Phi^{2} \pm 4 V R}, \\
R & =\sqrt{(\Phi \cos 2 \theta)^{2}+(\mu B)^{2}},
\end{aligned}
$$

where $V=G_{\mathrm{F}} n / \sqrt{2}$ as in Sec II. The time evolution of the wave function is described by the formula,

$$
\begin{aligned}
\Psi(t)= & \sum_{\zeta= \pm 1}\left[\left(U_{\zeta} \otimes U_{\zeta}^{\dagger}\right) \exp \left(-\mathrm{i} \mathcal{E}_{\zeta} t\right)\right. \\
& \left.+\left(V_{\zeta} \otimes V_{\zeta}^{\dagger}\right) \exp \left(\mathrm{i} \mathcal{E}_{\zeta} t\right)\right] \Psi_{0}
\end{aligned}
$$

where $U_{\zeta}$ and $V_{\zeta}$ are the eigenvectors of the Hamiltonian (4.5), given as

$$
U_{\zeta}=\frac{1}{N_{\zeta}}\left(\begin{array}{c}
-x_{\zeta} \\
-y_{\zeta} \\
1 \\
-z_{\zeta}
\end{array}\right), \quad V_{\zeta}=\frac{1}{N_{\zeta}}\left(\begin{array}{c}
-y_{\zeta} \\
x_{\zeta} \\
z_{\zeta} \\
1
\end{array}\right)
$$

where

$$
\begin{aligned}
x_{\zeta}= & \frac{\mu B\left(\mathcal{E}_{\zeta}+\Phi\right)}{\Sigma_{\zeta}} V \sin 2 \theta \\
y_{\zeta}= & \frac{\mu B}{\mathcal{E}_{\zeta}+\Phi-V \cos 2 \theta / 2}\left[1+\frac{\left(\mathcal{E}_{\zeta}+\Phi\right)}{2 \Sigma_{\zeta}} V^{2} \sin ^{2}(2 \theta)\right] \\
z_{\zeta}= & \frac{V \sin 2 \theta}{2\left(\mathcal{E}_{\zeta}+\Phi+V \cos 2 \theta / 2\right)}\left[1+\frac{2(\mu B)^{2}\left(\mathcal{E}_{\zeta}+\Phi\right)}{\Sigma_{\zeta}}\right] \\
\Sigma_{\zeta}= & \frac{V}{2}\left[2 \mathcal{E}_{\zeta}\left(\mathcal{E}_{\zeta}+\Phi\right)-V^{2} / 2+\Phi V \cos 2 \theta\right] \cos 2 \theta \\
& +\zeta R V\left(\mathcal{E}_{\zeta}+\Phi-V \cos 2 \theta / 2\right) .
\end{aligned}
$$

The normalization coefficient $N_{\zeta}$ in Eq. (4.8) is given by $N_{\zeta}=\sqrt{1+x_{\zeta}^{2}+y_{\zeta}^{2}+z_{\zeta}^{2}}$.

Proceeding along the same lines as in Sec. II, we obtain from Eqs. (3.1) and (4.7)-(4.9) the probability of the process $\nu_{\beta}^{\mathrm{L}} \rightarrow \nu_{\alpha}^{\mathrm{R}}$ as,

$$
\begin{aligned}
P_{\nu_{\beta}^{\mathrm{L}} \rightarrow \nu_{\alpha}^{\mathrm{R}}}(t)= & {\left[C_{+} \cos \left(\mathcal{E}_{+} t\right)+C_{-} \cos \left(\mathcal{E}_{-} t\right)\right]^{2} } \\
& +\left[S_{+} \sin \left(\mathcal{E}_{+} t\right)+S_{-} \sin \left(\mathcal{E}_{-} t\right)\right]^{2},
\end{aligned}
$$

where

$$
\begin{aligned}
C_{\zeta} & =-\frac{1}{N_{\zeta}^{2}}\left[\sin 2 \theta\left(x_{\zeta}+y_{\zeta} z_{\zeta}\right)+\cos 2 \theta\left(y_{\zeta}-x_{\zeta} z_{\zeta}\right)\right] \\
S_{\zeta} & =\frac{1}{N_{\zeta}^{2}}\left(y_{\zeta}+x_{\zeta} z_{\zeta}\right)
\end{aligned}
$$

Consistently with Eq. (3.2), we have taken the initial wave function as

$$
\Psi_{0}^{\mathrm{T}}=(\sin \theta, \cos \theta, 0,0) .
$$

With help of Eqs. (4.9) and (4.11) it is easy to check that $C_{+}+C_{-}=0$ guaranteeing $P(0)=0$.

Note that formally Eq. (4.10) corresponds to the transitions $\nu_{\beta}^{\mathrm{L}} \rightarrow \nu_{\alpha}^{\mathrm{R}}$. However, virtually it describes oscillations between active neutrinos $\nu_{\beta}^{\mathrm{L}} \leftrightarrow\left(\nu_{\alpha}^{\mathrm{L}}\right)^{c}$ since $\nu_{\alpha}^{\mathrm{R}}=\left(\nu_{\alpha}^{\mathrm{L}}\right)^{c}$ for Majorana particles.

As in the previous case of Eq. (2.21), Eq. (4.10) can be treated analytically for relatively small values of the effective potential $V$. The ensuing envelope functions $P_{u, d}=P_{0} \pm \sqrt{P_{c}^{2}+P_{s}^{2}}$ depend on the coefficients $C_{\zeta}$ and $S_{\zeta}$ in the same way as in Eq. (2.24). The transition probabilities at various values of the matter density and the magnetic field are presented in Fig. 4

Despite the formal similarity between Dirac and Majorana transition probabilities (see Eqs. (2.22) and (4.11) the actual dynamics is quite different in these two cases, as one can see by comparing Figs. 11(d)-3(d) and Fig. 4 , panels (b), (d) and (f). In particular, in the Majorana case $P_{u}^{(\max )}=4\left|C_{+} C_{-}\right|$for arbitrary $B$, to be compared with Eq. (2.26), while the function $P_{0}^{(\max )}$ has the same form as in the Dirac case given in Eq. (2.27). In contrast with the Dirac case, the averaged transition probability does not achieve its maximal value at some moderate magnetic field $B_{\max }$ value, but both $P_{u}^{\max }$ and $P_{0}^{\max }$ are monotonically increasing functions of the strength of the magnetic field with 1 and $1 / 2$ as their asymptotic values, respectively. We can understand this behavior when we recall that, at $\mu B \gg \max (\Phi, V)$, the effective Hamiltonian Eq. (4.5) becomes

$$
H_{\infty}=\mathrm{i} \mu B \gamma^{2}, \quad \mathrm{i} \gamma^{2}=\left(\begin{array}{cccc}
0 & 0 & 0 & 1 \\
0 & 0 & -1 & 0 \\
0 & -1 & 0 & 0 \\
1 & 0 & 0 & 0
\end{array}\right)
$$

The Schrödinger equation with the effective Hamiltonian (4.13) has the formal solution

$$
\begin{aligned}
\Psi(t) & =\exp \left(-\mathrm{i} H_{\infty} t\right) \Psi(0) \\
& =\left[\cos (\mu B t)+\gamma^{2} \sin (\mu B t)\right] \Psi(0) .
\end{aligned}
$$

Using Eqs. (3.1), (4.12) and (4.14) we then immediately arrive to the following expression for the transition probability, $P(t)=\left|\nu_{\alpha}^{\mathrm{R}}\right|^{2}=\sin ^{2}(\mu B t)$, which explains the behavior of the function $P_{u}^{(\max )}$ at strong magnetic fields. Note that the analogous result was also obtained in Ref. [22].

Finally, it is worth of noticing that in contrast to the Dirac case, the behavior of the transition probability in the Majorana case is qualitatively similar for different matter densities and different magnetic fields [see Fig. 4, panels (a), (c), and (e)].

The problem of Majorana neutrinos' spin-flavor oscillations was studied in Refs. [8, 9] with help of numerical codes. For example, in Ref. [8] the evolution equation for three neutrino flavors propagating inside a presupernova star with zero metallicity, e.g., corresponding 


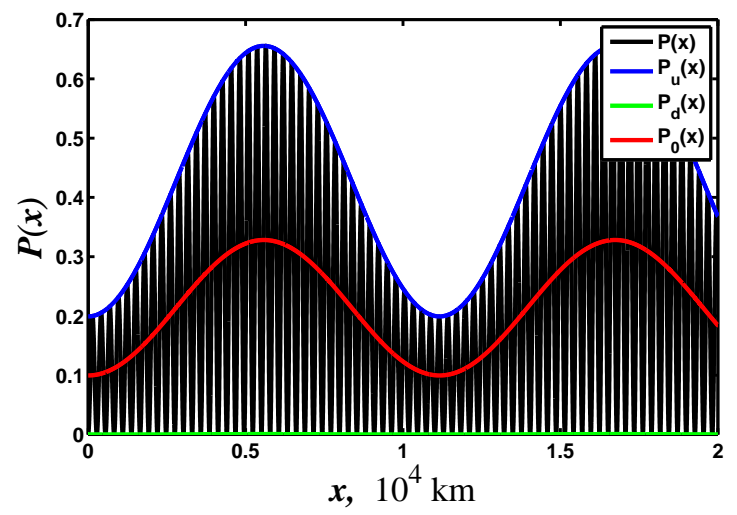

(a)

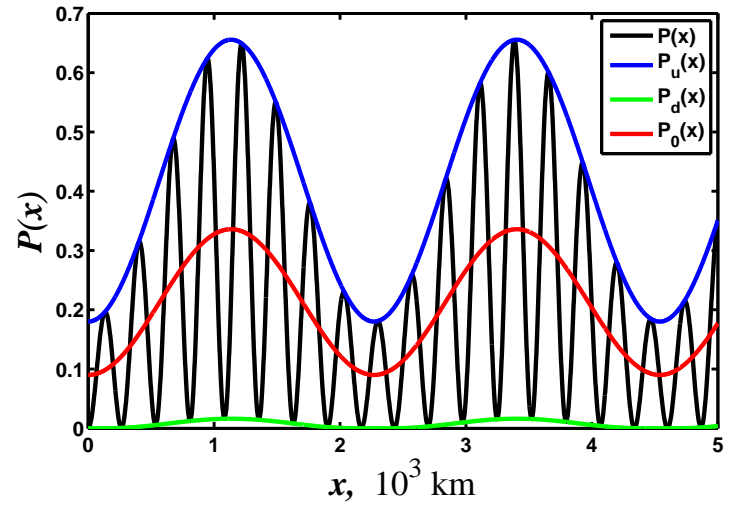

(c)

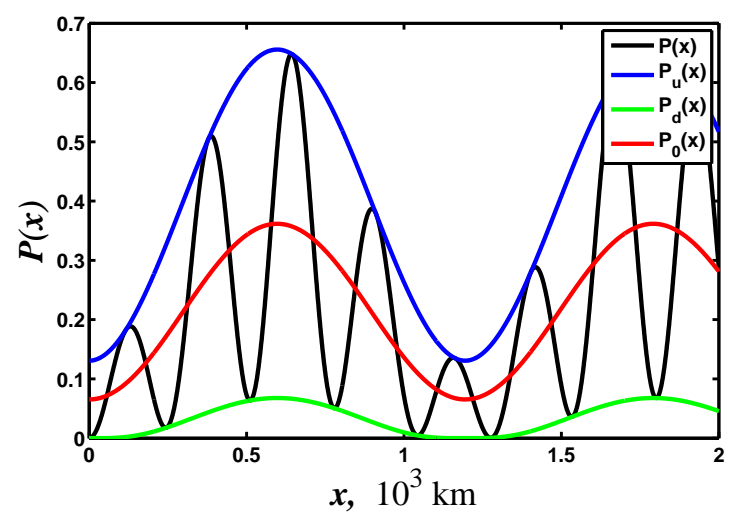

(e)

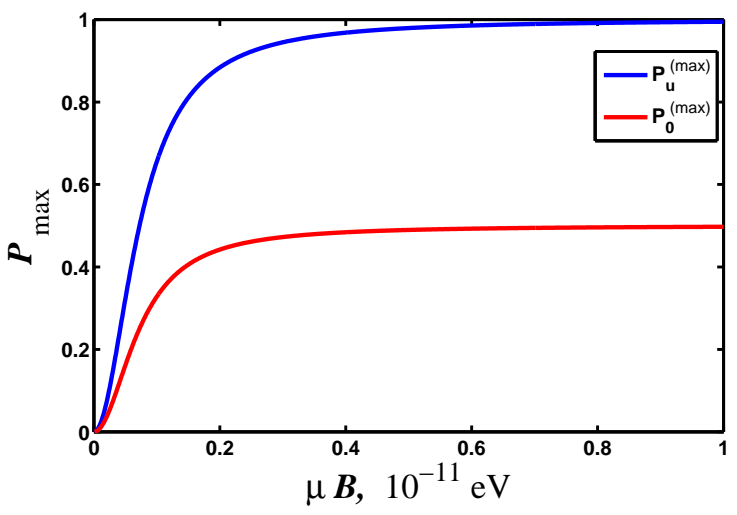

(b)

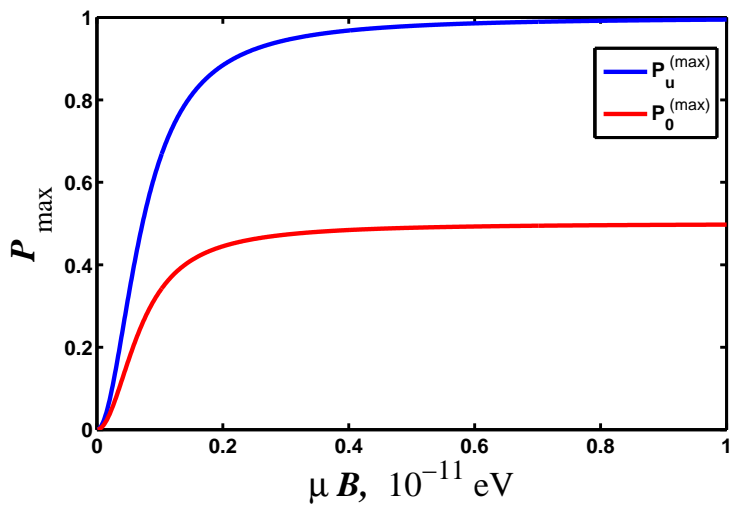

(d)

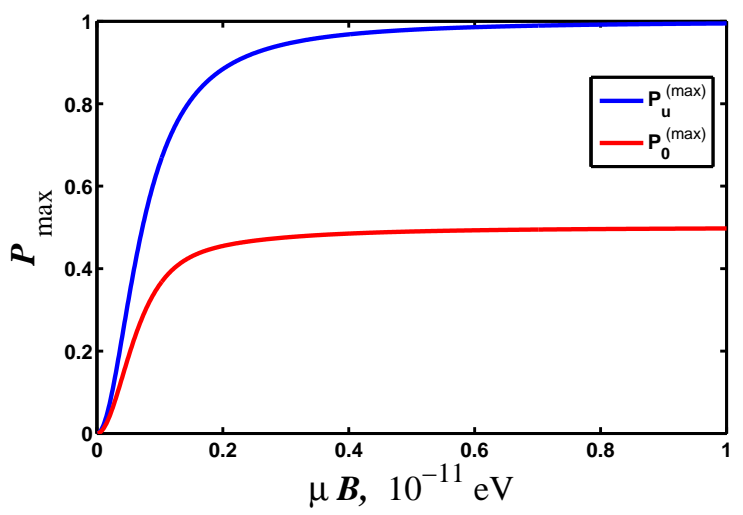

(f)

FIG. 4: (a), (c) and (e) The transition probability versus the distance passed by a neutrino beam. The neutrino parameters have the same values as in Figs. 1.3 $E_{\nu}=10 \mathrm{MeV}, \delta m^{2}=8 \times 10^{-5} \mathrm{eV}^{2}$ and $\theta=0.6$. The magnetic energy is equal to $\mu B=10^{-12} \mathrm{eV}$. The black line is the function $P(x)$, the blue and green lines are the envelope functions $P_{u, d}(x)$ and the red line is the averaged transition probability $P_{0}(x)$. (b), (d) and (f) The dependence of the maximal values of the functions $P(x)$ and $P_{0}(x)$, blue and red lines respectively, on the magnetic energy for the given density. Panels (a) and (b) correspond to the matter density $\rho=10 \mathrm{~g} / \mathrm{cc},(\mathrm{c})$ and (d) - to $\rho=50 \mathrm{~g} / \mathrm{cc}$ and (e) and (f) - to $\rho=100 \mathrm{~g} / \mathrm{cc}$.

to W02Z model [21], was solved for the realistic matter and magnetic field profiles. Although our analytical transition probability formula (4.10) is valid only for the constant matter density and magnetic field strength, it is interesting to compare our results with the numerical simulations of Ref. [8]. In those calculations the authors used magnetic fields $B \sim 10^{10} \mathrm{G}$ and magnetic moments $\sim 10^{-12} \mu_{\mathrm{B}}$ that give us the magnetic energy 
$\mu B \sim 10^{-11} \mathrm{eV}$. This value is the maximal magnetic energy used in our work.

It was found in Ref. [8] that spin-flavor conversion is practically adiabatic for low-energy neutrinos corresponding to $E_{\nu} \sim 5 \mathrm{MeV}$ inside the region where $Y_{e} \approx 0.5$ and the averaged transition probability for the channel $\nu_{\mu} \rightarrow \bar{\nu}_{e}$ is close 0.5 . This big transition probability is due to the RSF-H and RSF-L resonances at the distance $\approx 0.01 R_{\odot}$. Even so that we study two neutrino oscillations scheme, we obtained the analogous behavior of $P_{0}^{(\max )}$ (see Fig. 44). However, in our case this big transition probability is due to the presence of the strong magnetic field (see Refs. [10, 22]). We cannot compare our transition probability formula (4.10) with the results of Ref. 8] for higher energies, $E_{\nu}>25 \mathrm{MeV}$, since spinflavor oscillations become strongly nonadiabatic for these kinds of energies and one has to take into account the coordinate dependence of the matter density which should decrease with radius as $1 / r^{3}[32$.

\section{SUMMARY}

We have studied in this work the propagation of massive flavor-mixed Dirac and Majorana particles in matter under the influence of an external magnetic field in an approach based on relativistic quantum mechanics. The magnetic moment matrix is assumed to be both in the Dirac and Majorana case nondiagonal in the mass eigenstate basis. Starting with Lorentz invariant wave equations, the interactions of neutrinos with matter and magnetic fields included, we derived the effective Hamiltonians and solve the evolution equations for the electrically neutral matter with $n_{n}=n_{p}$. We then found the probabilities for the spin-flavor oscillation $\nu_{\beta}^{\mathrm{L}} \rightarrow \nu_{\alpha}^{\mathrm{R}}$ $\left(\nu_{\beta}^{\mathrm{L}} \rightarrow\left(\nu_{\alpha}^{\mathrm{L}}\right)^{c}\right.$ for Majorana neutrinos) and examined their dependence on the matter density and the strength of the magnetic field.

We also studied in our approach the evolution of mixed massive Majorana neutrinos in vacuum, which is an exactly solvable problem. We derived the wave functions of the neutrinos and the transition probabilities for the process $\nu_{\beta} \rightarrow \nu_{\alpha}$. We included the terms quadratic in neutrino masses and discussed the origin of these corrections of the standard formula for the probability.

These corrections to the Pontecorvo formula, see Eq. (3.12), are rapidly oscillating functions suppressed by the ratio $m_{a} /|\mathbf{k}|$, which is small for the relativistic neutrinos. It was revealed in our previous works [11, 12, 13, 14] that the analogous terms originate from the accurate account of the Lorentz invariance in the study of oscillations of Dirac neutrinos. It is also known that in describing of evolution of Majorana neutrinos in vacuum the transitions of the types $\nu_{\beta}^{\mathrm{L}} \rightarrow \nu_{\alpha}^{\mathrm{L}}$ and $\nu_{\beta}^{\mathrm{L}} \rightarrow\left(\nu_{\alpha}^{\mathrm{L}}\right)^{c}$ are possible [33], with the transition probability of the latter case being suppressed by the factor $m_{a} /|\mathbf{k}|$. The transitions $\nu_{\beta}^{\mathrm{L}} \rightarrow\left(\nu_{\alpha}^{\mathrm{L}}\right)^{c}$ can be interpreted as neutrinoto-antineutrino oscillations and manifest in the possible neutrinoless double beta decay [34]. In our work on the basis of the relativistic quantum mechanics we derived the transition probability for neutrino-to-antineutrino oscillations which is consistent with the results of Ref. [33].

Our results can be used for analyzing the behavior of neutrinos in supernovae, in particular in the so-called zero metallicity presupernovae, where the condition $n_{n}=$ $n_{p}$ is fulfilled [21] outside the $\mathrm{Si}+\mathrm{O}$ layer. It is known that these stars can possess very strong magnetic fields, up to $10^{15} \mathrm{G}$, or even stronger [35].

We found that large rates of transitions that change neutrino flavors and chiralities are possible both in the Dirac and Majorana cases with a typical oscillation lengths of the order of $10^{3}-10^{4} \mathrm{~km}$ for matter densities of the order of $10-100 \mathrm{~g} / \mathrm{cc}$, for typical supernova neutrino energies of $10 \mathrm{MeV}$ and for $\mu B$ of the order of $10^{-13}-10^{-12} \mathrm{eV}$. The parameters of a shock wave, the density and width, can vary in a rather wide range, but typically they fit with the parameter values we used in our analysis [36]. In particular, the width of the shock wave can be of about the same size as the oscillation length of the spin-flavor oscillations. It would thus be possible that the transition probability achieves its maximum value (can be as high as 0.8 in some cases) when neutrinos pass through the shock wave on their way towards the outer layers of the star. If the flight distance were many oscillation lengths, the probability would be averaged to a smaller value. The results of our work can have the implication to the r-process nucleosynthesis since it is sensitive to the amount of neutrinos of various species emitted in a supernova [37].

In the Dirac case the maximum value of the transition probability is achieved for a specific value of $\mu B$, while in the Majorana case the transition probability can be large for a wide range of $\mu B$ values. Supposing that the neutrino magnetic moment is $3 \times 10^{-12} \mu_{\mathrm{B}}$, allowed by the present astrophysical and cosmological data [38], the largest transition in the Dirac case would occur when the strength of the magnetic field is in the range $10^{8}-$ $10^{9} \mathrm{G}$. Although a magnetic field on the surface of a neutron star is typically stronger, $10^{12}-10^{13} \mathrm{G}$, at outer parts of the envelope it may have a suitable value for a large transition to take place. The difference between the dynamics of Dirac and Majorana particles under the influence of the same external fields can be a smoking gun to reveal the nature of neutrinos.

Note that in the majority of the previous works devoted to the spin-flavor oscillations the analytical transition probability formulae were obtained only for Majorana neutrinos. The case of Dirac particles was studied only in connection with small off-diagonal magnetic moments. Our work studies the opposite situation of big off-diagonal elements of the magnetic moments matrix. The effect of the appearance of the big transition probability at moderate magnetic field strength has never been described earlier. As for Majorana neutrinos the enhancement of the transition probability in strong magnetic fields is in agreement with the previous stud- 
ies [10,22]. Note that spin-flavor oscillations of Majorana neutrinos can be also resonantly enhanced in the moderate magnetic field, see Ref. [5]. However this effect does not happen in isoscalar matter with $n_{e}=n_{p}=n_{n}$. The condition for the resonant spin-flavor precession reads [7], $G_{\mathrm{F}}\left(n_{e}-n_{n}\right)= \pm \delta m^{2} \cos 2 \theta / 4 k$, which is not fulfilled in isoscalar matter.

The obtained analytical formulae for the transition probabilities, Eqs. (2.21) and (4.10), are valid in the important case when $Y_{e}=0.5$, which is realized in the zero metallicity presupernova stars. These kinds of stars were quite common for early stages of the galaxies formation. We obtained that spin-flavor oscillations of Dirac neutrinos can be strongly influenced by the moderate magnetic field of a neutron star and the big transition probability can exist not only for Majorana neutrinos [8, 9]. Thus spin-flavor oscillations of Dirac neutrinos can significantly change the relic supernova neutrino background [39]. Although now no signal of relic neutrinos was observed [40], there are still some efforts to calculate the flux of relic $\bar{\nu}_{e}$ for the KamLAND and Super-Kamiokande detectors [41]. The reliable simulation of the supernova explosion does not exist yet. For example, the propagation of a shock wave can significantly change magnitude and shape of the magnetic field which are very important in our calculations. Nevertheless it is believed that the future galactic supernova neutrino burst might give some clues to the physics of relic supernova neutrinos [9].

\section{Acknowledgments}

The work has been supported by the Academy of Finland under Contracts Nos. 108875 and 104915. MD is thankful to the Conicyt (Chile), Programa Bicentenario PSD-91-2006, for the grant as well as to Victor Semikoz (IZMIRAN) for helpful discussions and to the anonymous referee for valuable comments.
[1] A. Cisneros, Astrophys. Space Sci. 10, 87 (1971); M. B. Voloshin, M. I. Vysotskiü, and L. B. Okun', Sov. Phys. JETP 64, 446 (1986).

[2] J. Schechter and J. W. F. Valle, Phys. Rev. D 24, 1883 (1981).

[3] C.-S. Lim and W. J. Marciano, Phys. Rev. D 37, 1368 (1988).

[4] E. Kh. Akhmedov, Sov. J. Nucl. Phys. 48, 382 (1988).

[5] J. Pulido, Phys. Rep. 211, 167 (1992); E. Kh. Akhmedov, S. T. Petcov, and A. Yu. Smirnov, Phys. Rev. D 48, 2167 (1993), hep-ph/9301211; A. B. Balantekin and P. Loreti, Phys. Rev. D 48, 5496 (1993); J. Barranco et al., Phys. Rev. D 66, 093009 (2002), hep-ph/0207326; A. Friedland and A. Gruzinov, Astropart. Phys. 19, 575 (2003), hepph/0202095; M. Picariello, et al., JHEP 11, 055 (2007), 0705.4070 [hep-ph].

[6] E. Kh. Akhmedov and J. Pulido, Phys. Lett. B 553, 7 (2003), hep-ph/0209192; A. B. Balantekin and C. Volpe, Phys. Rev. D 72, 033008 (2005), hep-ph/0411148.

[7] H. Nunokawa, Y.-Z. Qian, and G. M. Fuller, Phys. Rev. D 55, 3265 (1997), astro-ph/9610209; H. Nunokawa, R. Tomas, and J. W. F. Valle, Astropart. Phys. 11, 317 (1999), astro-ph/9811181; E. Kh. Akhmedov and T. Fukuyama, JCAP 0312, 007 (2003), hep-ph/0310119.

[8] S. Ando and K. Sato, Phys. Rev. D 68, 023003 (2003), hep-ph/0305052.

[9] S. Ando and K. Sato, JCAP 0310, 001 (2003), hep$\mathrm{ph} / 0309060$.

[10] C. Giunti and A. Studenikin, 0812.3646 [hep-ph] (2008).

[11] M. Dvornikov, Phys. Lett. B 610, 262 (2005), hepph/0411101; in Proceedings of the IPM school and conference on Lepton and Hadron Physics, Tehran, 2006, ed. by Y. Farzan, eConf C0605151 (2007), hep-ph/0609139; Phys. Atom. Nucl. 72, 116 (2009), hep-ph/0610047.

[12] M. Dvornikov, Eur. Phys. J. C 47, 437 (2006), hepph/0601156; J. Phys. Conf. Ser. 110, 082005 (2008), 0708.2975 [hep-ph].
[13] M. Dvornikov and J. Maalampi, Phys. Lett. B 657, 217 (2007); hep-ph/0701209.

[14] M. Dvornikov, J. Phys. G 35, 025003 (2008); 0708.2328 [hep-ph].

[15] S. R. Elliott and J. Engel, J. Phys. G 30, R183 (2004), hep-ph/0405078.

[16] C. Giunti and C. W. Kim, Fundamentals of Neutrino Physics and Astrophysics (Oxford Univ. Press, Oxford, 2007), pp. 320-321.

[17] L. Wolfenstein, Phys. Rev. D 17, 2369 (1978); M. Dvornikov and A. Studenikin, JHEP 09, 016 (2002), hep-ph/0202113.

[18] N. F. Bell, et al., Phys. Rev. Lett. 95, 151802 (2005); hep-ph/0504134.

[19] E. V. Arbuzova, A. E. Lobanov, and E. M. Murchikova, Phys. Atom. Nucl. 72, 141 (2009), 0711.2649 [hep-ph].

[20] A. Studenikin and A. Ternov, Phys. Lett. B 608, 107 (2005), hep-ph/0412408; A. E. Lobanov, Phys. Lett. B 619, 136 (2005), hep-ph/0506007.

[21] S. E. Woosley, A. Heger and T. A. Weaver, Rev. Mod. Phys. 74, 1015 (2002).

[22] G. G. Likhachev and A. I. Studenikin, JETP 81, 419 (1995).

[23] S. Kawagoe, et al., J. Phys. Conf. Ser. 39, 294 (2006).

[24] M. Fukugita and T. Yanagida, Physics of Neutrinos and Applications to Astrophysics (Springer, Berlin, 2003), pp. 292-296.

[25] K. M. Case, Phys. Rev. 107, 307 (1957).

[26] V. B. Berestetskiǔ, E. M. Lifschitz, and L. P. Pitaevskiŭ, Quantum Electrodynamics (Moscow, Nauka, 1989), pp. 108-112.

[27] See pp. 137-141 in Ref. 26].

[28] M. Blasone and G. Vitiello, Ann. Phys. 244, 283 (1995), Erratum ibid. 249 (1996) 363, hep-ph/9501263.

[29] D. Giulini, et al., Decoherence and the Appearence of a Classical World in Quantum Theory (Springer-Verlag, Berlin, 1996). 
[30] See pp. 477-478 in Ref. 24].

[31] S. Pastor, Master's Thesis (University of Valencia, 1996).

[32] M. Kachelrieß, et al., Phys. Rev. D 65, 073016 (2002), hep-ph/0108100.

[33] I. Yu. Kobzarev, et al., Sov. J. Nucl. Phys. 35, 708 (1982).

[34] G. Barenboim, et al., Phys. Lett. B 537, 227 (2002), hep$\mathrm{ph} / 0203261$.

[35] D. J. Price and S. Rosswog, Science 312, 719 (2006).

[36] A. Burrows, K. Klein, and R. Gandhi, Phys. Rev. D 45, 3361 (1992); R. Tomàs, et al., JCAP 0409, 015 (2004), astro-ph/0407132.

[37] A. B. Balantekin and G. M. Fuller, J. Phys. G 29, 2513 (2003), astro-ph/0309519.

[38] G. G. Raffelt, Phys. Rev. Lett. 64, 2856 (1990).

[39] See pp. 515-516 in Ref. 16].

[40] M. Malek, et al., Phys. Rev. Lett. 90, 061101 (2003), hep-ex/0209028.

[41] L. E. Strigari, et al., JCAP 0403, 007 (2004), astro$\mathrm{ph} / 0312346$. 